\title{
Real-Time Visualization of Large-Scale Geological Models with Nonlinear Feature-Preserving Levels of Detail
}

\author{
Ronell Sicat, Mohamed Ibrahim, Amani Ageeli, Florian Mannuss, Peter Rautek, and Markus Hadwiger
}

\begin{abstract}
The rapidly growing size and complexity of 3D geological models has increased the need for level-of-detail techniques and compact encodings to facilitate interactive visualization. For large-scale hexahedral meshes, state-of-the-art approaches often employ wavelet schemes for level of detail as well as for data compression. Here, wavelet transforms serve two purposes: (1) they achieve substantial compression for data reduction; and (2) the multiresolution encoding provides levels of detail for visualization. However, in coarser detail levels, important geometric features, such as geological faults, often get too smoothed out or lost, due to linear translationinvariant filtering. The same is true for attribute features, such as discontinuities in porosity or permeability. We present a novel, integrated approach addressing both purposes above, while preserving critical data features of both model geometry and its attributes. Our first major contribution is that we completely decouple the computation of levels of detail from data compression, and perform nonlinear filtering in a high-dimensional data space jointly representing the geological model geometry with its attributes. Computing detail levels in this space enables us to jointly preserve features in both geometry and attributes. While designed in a general way, our framework specifically employs joint bilateral filters, computed efficiently on a high-dimensional permutohedral grid. For data compression, after the computation of all detail levels, each level is separately encoded with a standard wavelet transform. Our second major contribution is a compact GPU data structure for the encoded mesh and attributes that enables direct real-time GPU visualization without prior decoding.
\end{abstract}

Index Terms-Geological models, structured hexahedral meshes, multiresolution representations, interactive visualization.

\section{INTRODUCTION}

I $\mathrm{N}$ recent years, the complexity and size of generated and simulated geological models has increased dramatically. Interactive visualization of large-scale geological models is still a very challenging task, leading to a need for novel scalable and interactive visualization techniques. Standard approaches for interactively visualizing large-scale spatial data rely on multiresolution representations and level-ofdetail approaches [1], [2], [3], [4], [5]. However, state-ofthe-art techniques for large-scale hexahedral meshes with attributes still face significant problems with respect to preserving the fine details and discontinuities in geological models, as illustrated in Fig. 1. Most techniques compute increasingly low-pass filtered and downsampled versions of the original data. Low-pass filtering helps avoid aliasing artifacts from undersampling, while downsampling helps speed up level-of-detail visualization by decreasing the amount of data that needs to be processed.

One fundamental problem of standard level-of-detail approaches is that low-pass filtering is performed with linear translation-invariant (LTI) filters. This means that in coarse detail levels, original data values are replaced by fewer values computed by averaging the original data according to weights that do not take all data properties into account. Linear filtering is extremely efficient, but it smoothes out discontinuities. This often leads to important features being lost or misrepresented in coarse detail levels.

- R. Sicat, M. Ibrahim, A. Ageeli, P. Rautek, and M. Hadwiger are with King Abdullah University of Science and Technology.

E-mail: ronell.sicat@kaust.edu.sa

- F. Mannuss is with Saudi Aramco.

Manuscript received January 31, 2021; revised October 07, 2021.
Moreover, transition artifacts between levels can become very noticeable when the weighted average differs significantly from the original data values contributing to the average [5], [6], [7]. Furthermore, discontinuities often shift in spatial location when transitioning between resolution levels, because the sample positions change significantly. All of these problems are not only disturbing visually, but can also lead to analysis errors when the full data resolution is not inspected everywhere or misunderstood.

For rendering 3D geological data, the problems listed above can result in the undesirable disappearance or degradation of important mesh discontinuities, in particular geological faults. The loss of important attribute discontinuities when transitioning to coarser resolution levels can become particularly dangerous for analysis due to the high number of different attribute channels, such as porosity and permeability, usually associated with geological data.

A recent multiresolution representation for geological models, HexaShrink [8], is able to better maintain geometric discontinuities across resolution levels by adaptively subsampling mesh vertices, i.e., prioritizing vertices that belong to geological faults. However, its wavelet-based encoding coupled with its choice of using low-frequency subbands or approximation coefficients as low-resolution versions of the attribute data oversmoothes attribute edges, as shown in Fig. 1 (d).

In typical wavelet-based multiresolution representations, this occurs because the wavelet's scaling function used to compute approximation coefficients has a similar effect as the LTI filtering in simpler anti-aliasing low-pass filters. They may also lead to oversmoothing of features in the coarse levels, especially along edges. In the case of Hex- 


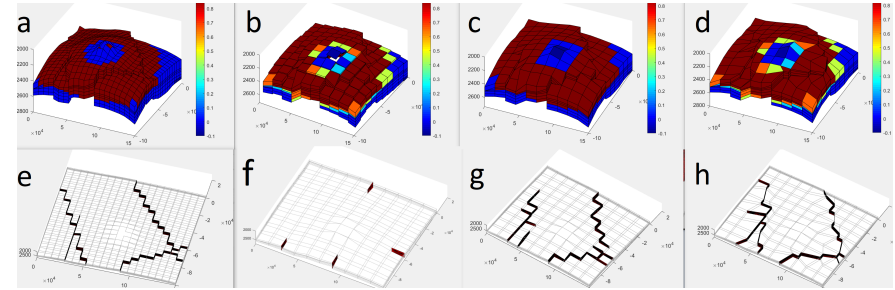

Fig. 1. Corner-point geological models containing attribute discontinuities (top) and geological faults (bottom). From the original data $(a, e)$ a low resolution version is computed using different methods on corner-point data: $(b, f)$ box filtering followed by subsampling introduces incorrect attributes along discontinuities (b) and removes faults (f); (c,g) subsampling only leads to aliasing artifacts such as skipped faults $(\mathrm{g})$; (d,h) HexaShrink [8] reduces mesh artifacts (h) but introduces attribute artifacts (d), similar to those introduced by box filtering (b).

aShrink, for instance, the use of Haar wavelets for encoding attribute data is equivalent to using a box or averaging lowpass filter (Fig. 1 (b)), which can lead to artifacts along sharp edges or discontinuities. Additionally, computing the multiresolution representation of the mesh geometry separately and independently from the scalar attributes may lose the spatial alignment between them in some regions. For structured meshes in particular, this happens when the mesh is subsampled adaptively instead of regularly (by skipping odd or even samples), while the attribute downsampling is not adapted accordingly. HexaShrink potentially misaligns the spatial locations of hexahedral cells from the original locations of important attribute discontinuities.

Contributions. In order to address these problems, we present a novel framework with the following main contributions. (1) An image-based level-of-detail computation for geological models that completely decouples the generation of a multiresolution hierarchy with progressively reduced detail from compressed encoding (Sec. 3). (2) Level-ofdetail computation by nonlinear feature-preserving filtering, using joint bilateral filters efficiently evaluated on a highdimensional permutohedral grid (Sec. 4). (3) A compact encoding of geological models in a custom GPU data structure (Sec. 5) that facilitates direct visualization on the GPU without prior decoding (Sec. 6).

By using an edge-preserving smoothing filter in our multiscale representation, the mixing of dissimilar regions in terms of both geometry and scalar attributes is avoided. This consequently avoids the introduction of undesirable new values in coarse levels and avoids the smoothing of important edges or features. Furthermore, we encode each scale or resolution level of our multiscale representation using its full hierarchy of wavelet transform coefficients. In contrast, standard wavelet-based techniques use a single hierarchy of wavelet transform coefficients to represent all resolution levels, and use approximation coefficients to represent coarse levels. Doing this allows us to leverage the benefits of wavelet-based coding, e.g., their sparsity and multiscale decomposition, without being tied to using approximation coefficients as coarse level representations, which we have seen to be problematic along edges.

\section{Related Work}

State-of-the-art geological model representations often focus on simplicity, data compression, or facilitating fast simulation without supporting interactive visualization of large- scale data. The corner-point grid data structure is a widely used industry standard for encoding geological models for reservoir and basin modeling and simulation [8], [9]. It stores the attributes and mesh vertices as a set of labeled one-dimensional lists of values encoded either in a standard ASCII file, e.g., the .GRDECL format, or in a binary file, e.g., the .EGRID format [9]. This simple approach is easy to parse and supports a wide range of structured grid variations, making it a common choice for geological model representation in various simulation architectures. Generalized prism grids (GPGs) [10] can represent pillarbased unstructured grids with arbitrary polyhedron cells, faults, degenerate layers, and overlapping pillars. GPGs focus on compatibility with different grid types, while supporting efficient simulation. Corner-point grids and GPGs can support most simulation tools due to their generality, but usually have very large storage requirements since they do not incorporate any compression. On their own, they also generally do not support scalable and interactive visualization. The HexaShrink [8] representation introduces separate wavelet-based encodings for the mesh and attribute data. While their encoding gives good compression, their wavelet representation can lead to loss of features in the attributes at coarse levels, as shown in Fig. 1. Focused on compressed data storage, it is unclear how the representation can be used for interactive visualization.

Multiresolution representations are typically employed by interactive large-scale visualization techniques to enable level-of-detail-based visualization. One interesting challenge posed by geological models is how to jointly compute and encode a multiresolution representation of the attribute and mesh data which are generally different in nature. That is, attributes are usually treated as $3 \mathrm{D}$ volumes and hexahedral meshes are treated as polygonal meshes.

Multiresolution 3D volumes typically use octrees [2] or 3D mipmaps [11], [12] in multiresolution or mixedresolution volume rendering [1], [3], [4], [13]. While these representations facilitate interactive multiresolution visualization, oftentimes the low-pass filtering used for antialiasing coupled with downsampling leads to undesirable loss of detail in coarse levels. Previous efforts have addressed this issue for large-scale volume data [5], [6], [14], [15] but these methods can be complex and do not necessarily apply directly to hexahedral meshes with attributes. Polygonal mesh representations encode the vertices and their connectivity. In GPU-based rendering, this information is typically encoded in arrays (vertex and index buffers) and directly uploaded to GPU memory for processing. Multiresolution representations are achieved by computing iteratively simplified meshes using various techniques [16], [17], [18] and encoding them in a data structure that fits the specific application. There has also been work in mesh simplification that maintains associated attributes [19], [20], [21]. While these approaches can be useful for our purposes, their GPU memory requirements can quickly increase. Furthermore, the decoupled mesh and attribute resolutions inhibit attribute-based visibility culling. Instead of using standard vertex/index list representations, we aim to explore compressed mesh and attribute representations that leverage the structure in corner-point grid data for a compact representation that still enables direct GPU-based level- 


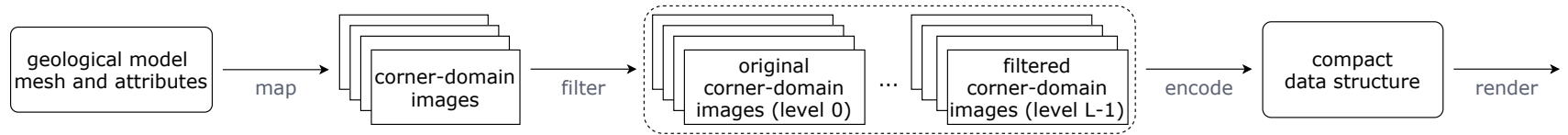

Fig. 2. Pipeline overview. We first map the geological model's per-vertex positions and per-cell attributes into a stack of images where each pixel is a per-corner position and attribute. We then compute a multiscale representation of the images (dashed box) using our feature-preserving smoothing filter. The pillar and $z$ coordinates and cell attributes are then encoded in our compact data structure which is directly used in rendering.

of-detail rendering. Our framework introduces an imagebased representation of the joint mesh and attribute data in a single domain, allowing us to jointly process them in computing our multiscale representation.

Wavelet-based representations are a specific kind of multiresolution representation, where a signal is decomposed into its low-frequency and high-frequency subbands. In multiresolution analysis using wavelet basis functions [22], these subbands are often called approximation and detail coefficients, respectively. Hierarchical wavelet transforms are often used as a multiresolution representation of large data [23], [24]. Typically, a discrete wavelet transform (DWT) is applied hierarchically on the original data, and processing can be performed directly on the wavelet transform coefficients. The inverse discrete wavelet transform (IDWT) is used to recover fine-level approximation coefficients from coarser level approximation and detail coefficients. The hierarchical nature of the wavelet coefficients, which decomposes the data into multiple resolution levels, is a natural fit for many level-of-detail applications. However, the traditional use of approximation coefficients as coarse data representations can pose problems, as illustrated in Fig. 1.

The sparsity of transform coefficients is usually leveraged for compact data encoding and compression. The most common wavelet encoding techniques used for image compression are EZW [25], EBCOT [26], SPIHT [27], WDR [28], and ASWDR [29]. These methods use embedded bit-plane coding of the coefficient magnitudes, allowing for progressive transmission of compressed images with quality scalability. They also leverage zerotrees, i.e., tree hierarchies of wavelet coefficients that correspond to the same spatial footprint that have similar significance [25], to minimize coding symbols. In our work, we will use zerotrees to refer to a tree hierarchy of coefficients whose values are all zero. Despite usually being lossy, these techniques offer very good tradeoffs between error and compression rates, with high perceptual quality [30]. However, these methods are typically difficult to parallelize and can have high-complexity and memory-inefficient decoding, which does not fit our shaderbased rendering architecture where compute and memory resources are limited (Sec. 6). In our framework, we use progressive resolution coding (PROGRES) [31], due to its low-complexity and memory-efficient decoding scheme that is well-suited for our GPU-based rendering approach.

\section{Method}

Our framework (Fig. 2) maps corner-point grid geological models to an image-based representation (see Figs. 3 and 4). We call the corresponding images geometry images. (This name is inspired by the work of Gu et al. [32], but our representation is not the same.) This approach unifies geometry and attributes in images $I$, and enables us to compute a multiscale hierarchy for level-of-detail rendering by evaluating nonlinear image filters in high-dimensional spaces. We compute $L$ detail levels, by applying a nonlinear feature-preserving smoothing filter $F_{\Omega}$ jointly to the mesh and attribute data, with $\Omega$ denoting filter parameters. Each level is computed from the input original data encoded in $I_{0}$, adapting $\Omega$ to apply more smoothing to coarser levels,

$$
I_{\ell}=F_{\Omega(\ell)}\left(I_{0}\right) \text {, with } \ell \in[1, L-1] .
$$

In each level $\ell>0$, similar or uniform regions are maximally smoothed, while dissimilar regions, e.g., discontinuities, are minimally smoothed, and therefore preserved. The smoothing filter's similarity criteria $\Omega$ are increasingly relaxed for coarser levels, resulting in progressively smoother versions of the original data in the multiscale representation.

Detail reduction vs. storage size. A crucial property of our approach is that we do not immediately reduce the sampling rate, i.e., the number of pixels, in coarser detail levels. We reduce the detail content without decreasing the sampling rate. This is similar to approaches such as base and detail layer decomposition for image manipulation [33]. We perform storage reduction in a completely separate stage, where each detail level is individually encoded using a wavelet transform. The fact that we have reduced the detail content naturally leads to smaller encodings. However, not reducing the sampling rate during filtering preserves the location of discontinuities more accurately. Moreover, since we work in high-dimensional spaces for filtering (combining geometric attributes with all other attributes), a similar approach solely based on wavelets would require highdimensional wavelet transforms. In contrast, common representations such as mipmaps, quadtrees, or wavelets, reduce the number of grid points in every level, e.g., by two along each dimension, reducing storage size jointly with detail content. This is simpler, but smoothes out features and/or moves their location. The latter is particularly disturbing in transitions between detail levels.

Geological layers. In addition to keeping the mesh and attribute resolutions constant across levels, we do not smooth or downsample along the vertical dimension, since geological data are highly anisotropic and contain much fewer layers than their resolution in $x$ and $y$. Furthermore, layers typically correspond to very different rock or basin types, for which it is also important to keep layers separate and preserve their number.

Nonlinear filters. Unlike the linear translation-invariant filtering used to compute coarse levels in standard representations, our nonlinear filtering preserves features better. In our framework, the filter $F$ is a black box, and therefore conceptually any linear or nonlinear filter can be used. We perform nonlinear feature-preserving filtering via joint bilateral filters, in order to preserve important geometric and attribute features in the data (Sec. 4). Our filters operate 


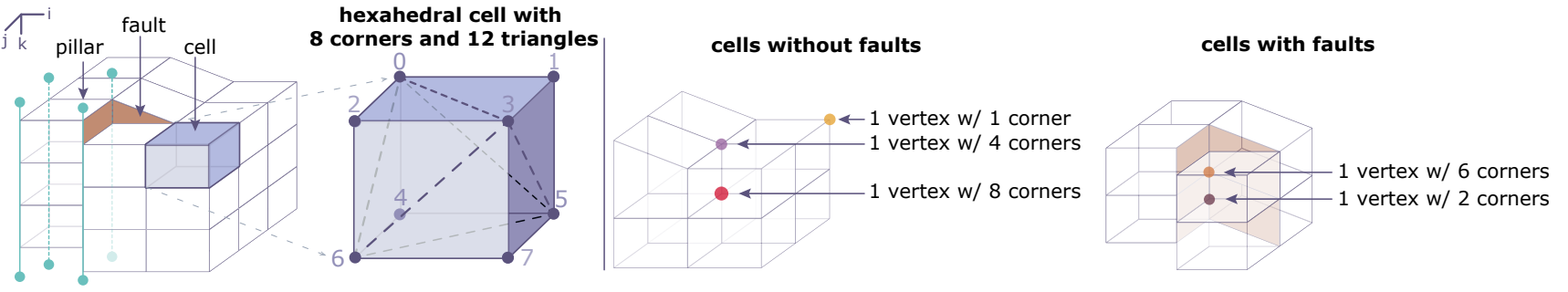

Fig. 3. Geological models. Left: Geological models are often represented using a corner-point grid, where corners of hexahedral cells lie on a set of vertical or inclined lines called pillars. Each hexahedral cell has 8 corners and 12 triangles with implicit topology. Right: Each mesh vertex can have between 1 and 8 corners, depending on the fault configuration of its $2 \times 2 \times 2$ topologically neighboring and surrounding cells.

in a high-dimensional domain. For efficient computation, we employ permutohedral grids [34].

Encoding and rendering. After level-of-detail computation, the next key component of our framework is a compact encoding of each detail level. Ideally, this encoding should be content- and feature-adaptive in order to reduce both storage and processing complexity for progressively coarser regions. Moreover, our encoding, together with custom GPU data structures, enables real-time visualization without performing decoding prior to rendering. We employ an adaptive resolution encoding, by applying wavelet transforms to our image-based level-of-detail representation, and render directly from the encoded data. Actual geometry is generated on-the-fly in GPU shaders. In terms of data storage, we use fewer bits to encode smooth or uniform regions, and more bits to encode high-detail regions. In terms of geometric complexity, uniform regions are visualized using fewer triangles (low geometric resolution), while high-detail regions are visualized with more triangles (high geometric resolution). See Secs. 5 and 6 .

\subsection{Data Description and Notation}

Geological models in the geosciences and oil and gas industry are often represented as hexahedral meshes with attributes. Each model typically consists of the mesh data to encode the geometry and topology of geological structures, and additional scalar-valued attributes for geophysical properties. We describe a geological model $G$ by its set of hexahedral cells $C$ that have mesh and attribute components (see Fig. 3). The mesh $M$ of the cells consists of a set of vertices $V$, and a set of triangle faces $T$. Each vertex $v \in V$ has a position $\mathrm{p}_{v} \in \mathbb{R}^{3}$. Each hexahedral cell $c \in C$ consists of 12 triangle faces defined by 12 vertex triplets, i.e., each triangle $t \in T$ is a vertex triplet $\left(v_{1}, v_{2}, v_{3}\right)$. All attributes are per-cell data. Each cell has a set of $m$ attribute scalars denoted by $\mathbf{a} \in \mathbb{R}^{m}$, or by $\mathbf{a}_{c}$ for a specific cell $c$. For instance, a model with scalar permeability and porosity attribute channels has $m=2$.

Our focus is on structured meshes where the set of hexahedral cells $C$ is topologically equivalent to a 3D Cartesian grid [8]. We denote a cell in a structured mesh with $C_{i} \times C_{j} \times C_{k}$ cells as $c_{i, j, k}$, with $i \in\left[0, C_{i}-1\right], j \in$ $\left[0, C_{j}-1\right], k \in\left[0, C_{k}-1\right]$. In the structured representation, a constant number of eight 3D positions are encoded per cell, corresponding to the eight corners of a hexahedron. Furthermore, connectivity information $T$ is implicit. That is, for each cell only the positions of its eight corner positions are needed to model the mesh. Each cell $c$ has eight cornerpoints or corners, $r_{c, n}$, with $n \in[0,7]$. Each corner has a geometric position $\mathrm{p}_{r_{c, n}} \in \mathbb{R}^{3}$. All topologically neighboring corners with the same position belong to the same vertex. Therefore, each vertex has $1 \leq h \leq 8$ corners, i.e., $\mathrm{p}_{r}=$ $\mathrm{p}_{v}, \forall r \in H_{v}$, where $H_{v}$ is the set of corners that belong to vertex $v . H_{v}$ implicitly encodes the fault configuration in the mesh. Excluding boundary corners, eight topologically neighboring corners in each $2 \times 2 \times 2$ cell neighborhood correspond to one vertex only if there are no faults. Fig. 3 shows example vertex and corner configurations.

\subsection{Corner-Point Grids}

Our work focuses on structured geological models, particularly corner-point grid data. In such data, corner-points lie along a set of straight lines called pillars (see Fig. 3). Pillars are coordinate lines that run from the top to the bottom of the geological model. Due to the constrained geometrical structure, the $(x, y, z)$ coordinates of corner points are efficiently encoded in a set of vertical or inclined pillars. Each pillar is compactly defined by its top $(x, y, z)_{\text {top }}$ start points and bottom $(x, y, z)_{b t m}$ end points. Given the $z$ coordinate of a corner, its $(x, y)$ coordinates can be linearly interpolated from its corresponding pillar information, removing the need to store all $(x, y)$ coordinates. Therefore, a geological model with $C_{i} \times C_{j} \times C_{k}$ cells and $m$ attributes per cell can be encoded via the following number of scalar values: $6 \cdot\left(C_{i}+1\right) \cdot\left(C_{j}+1\right)$ for pillar coordinates, i.e., $(x, y, z)_{\text {top }}$ and $(x, y, z)_{b t m}, 8 \cdot C_{i} \cdot C_{j} \cdot C_{k}$ for eight $z$ coordinates per cell (one per corner), and $m \cdot C_{i} \cdot C_{j} \cdot C_{k}$ for $m$ attributes per cell. This total number of scalars multiplied by the number of Bytes of each scalar, e.g., 4 Bytes for single precision floats, is the amount of memory needed to encode the data, i.e., its storage size. The corner-point grid is one of the industry standards for encoding geological models [8]. Its simplicity and generality make it suitable for a wide range of modeling, simulation, and visualization tools.

\subsection{Mapping Corner-Point Grids to Geometry Images}

A large part of our framework operates on images. We map the cells $C$ of a corner-point grid, with $C_{k}$ cells in the vertical direction, to a stack of $2 C_{k}$ geometry images $\left\{I_{g}\right\}$, with $g \in\left[0,2 C_{k}-1\right]$, as illustrated in Fig. 4. Each image $I_{g}$ has a resolution of $2 C_{i} \times 2 C_{j}$ pixels. In the image domain, filtering and encoding become much simpler than on the original geometry. During rendering, the image representation is decoded and mapped back on-the-fly to the corresponding geometry.

Each layer $k$ of cells maps to two consecutive images $I_{2 k}, I_{2 k+1}$. Specifically, each of the eight corners of cell $c_{i, j, k}$ maps to exactly one pixel. The top $2 \times 2$ corners map to image $I_{2 k}$, and the bottom $2 \times 2$ corners map to image $I_{2 k+1}$. All geometry images are multi-channel images, with each 


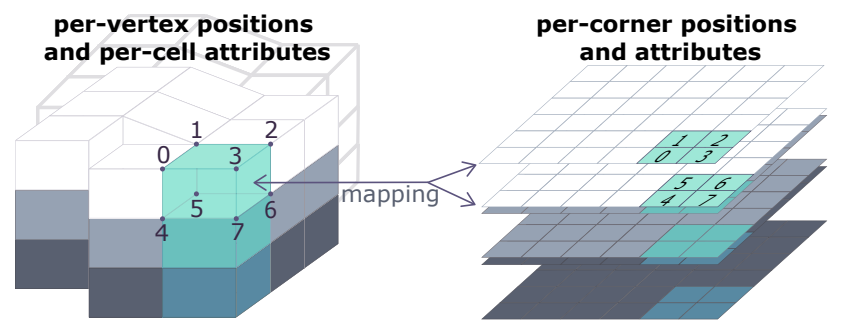

Fig. 4. Geometry images. We map per-vertex positions and per-cell attributes of a geological model (left) to and from a stack of geometry images (right) where each pixel is a per-corner position and attribute.

image comprising $3+m$ scalar channels, for $m$ attributes. Each pixel stores the corresponding 3D corner position $\mathrm{p}_{r} \in \mathbb{R}^{3}$, and all corner attributes $\mathbf{a}_{r} \in \mathbb{R}^{m}$. Thus, each pixel is $\in \mathbb{R}^{3+m}$.

The per-vertex positions are mapped to corner positions using $\mathrm{p}_{r}=\mathrm{p}_{v} \forall r \in H_{v}$. The per-cell attributes are mapped to corner attributes using $\mathbf{a}_{r}=\mathbf{a}_{c} / 8$. Therefore, a geological model with $C_{i} \times C_{j} \times C_{k}$ cells gets mapped to a stack of $2 C_{k}$ images $\left(I_{g}\right.$ with $\left.g \in\left[0,2 C_{k}-1\right]\right)$ with $2 C_{i} \times 2 C_{j}$ pixels and $(3+m)$ channels each. The mapping of cell data to the image stack puts the vertex- and cellbased information in a single corner-based domain in which we can operate as in regular multichannel images. In our framework, filtering the geological model is applied by filtering the stack of images $\left\{I_{g}\right\}$ (see Sec. 4). After filtering, we map the filtered image data back to vertex positions and cell attributes to get the resulting filtered model. To map back corner attributes $\mathbf{a}_{r}$ to cells, we use $\mathbf{a}_{c}=\sum_{i=0}^{7} \mathbf{a}_{r_{i}}$, where $r_{i}$ are the corners of cell $c$. The vertex positions are computed using $\mathrm{p}_{v}=\frac{1}{h} \sum_{i=1}^{h} \mathrm{p}_{r_{i}}, \forall r_{i} \in H_{v}$. In practice, we always store the eight corner $z$ coordinates per cell regardless of vertex information (e.g., we store eight duplicate $z$ coordinates for the eight corners of one vertex), in order to preserve the regular structure of the pillar-based data. Vertex information is implicit since it is only based on the groups of topologically neighboring corners, i.e., $H_{v}$. Therefore, we keep the vertex information intact via $\mathrm{p}_{r}=\mathrm{p}_{v}, \forall r \in H_{v}$. This conceptual mapping to vertices is important in preserving the geological fault information in the data after filtering the corner positions. In addition, we perform a post-filter operation on the $z$ coordinate channel to make sure that: (1) for every vertically neighboring cells denoted by $c_{i, j, k}$ (top cell) and $c_{i, j, k+1}$ (bottom cell), the bottom corner $z$ coordinates of the top cell should be equal to the top corner $z$ coordinates of the bottom cell (no vertical gaps between cells), and (2) along each pillar line, the $z$ coordinates of a top cell $c_{i, j, k}$ should be less than or equal to the $z$ coordinates of the cell below it $c_{i, j, k+1}$ ( $z$ coordinates along a pillar should be monotonously increasing from top to bottom). To satisfy condition (1), we set the $z$ coordinates of overlapping corners to their average value. To satisfy condition (2) along each pillar, $z$ coordinates of top cells that exceed the $z$ coordinates of the cells below them are set equal to the latter. These are typical approaches for fixing corner-point grid coordinates [9].

\section{Nonlinear Feature-Preserving Filtering}

The nonlinear feature-preserving smoothing filter $F_{\Omega}$ is used to compute the coarse levels in our multiscale rep- resentation. Since $F_{\Omega}$ operates in the image domain, we first map the original cells $C$, i.e., its vertex and attribute information, to the geometry image representation $\left\{I_{g}\right\}$ discussed above. The filter then computes $I_{\ell}=\left\{F_{\Omega(\ell)}\left(I_{0}\right)\right\}$, with $\ell \in[1, L-1]$ and $\Omega(\ell)$ comprising the filter parameters for level $\ell$. Our filter successively smoothes the imagebased representation of the original data $I_{0}$ while keeping important features. The feature-preserving property of the nonlinear filter avoids the mixing of dissimilar values along edges in the data, therefore avoiding feature degradation.

\subsection{Joint Bilateral Filtering}

In our framework, the filter $F_{\Omega}$ is a joint bilateral filter [35] applied in the joint spatial and attribute domain of $(3+$ $m$ ) dimensions, illustrated in Fig. 5. When filtering spatial coordinates of corners, we filter only the $z$ coordinates and keep the $(x, y)$ coordinates intact. We also do not filter the $(x, y, z)_{\text {top } \mid b t m}$ coordinates of the pillars. Filtering only the $z$ values means the corner point positions only move along the pillar lines. The pillar coordinates themselves are never filtered or downsampled, but are encoded compactly in the data structure as their size is very small compared to the whole dataset, i.e., only two $\left(C_{i}+1\right) \times\left(C_{j}+1\right)$ pixel images with $(x, y, z)$ values per pixel. Doing so allows us to always reconstruct the $(x, y)$ coordinates at any resolution we need, and is also helpful in minimizing seams between tiles of different resolution levels in mixed-resolution rendering.

We filter the $z$ coordinates using Gaussian weights based on spatial positions and attributes, altogether giving $3+m$ dimensions. The filtered coordinate $z_{\mathrm{p}}^{\prime} \in \mathbb{R}$ at spatial position $\mathrm{p} \in \mathbb{R}^{3}$ of a corner-point, with $z$ coordinates $\left\{z_{\mathrm{q}}\right\}_{\mathrm{q} \in S}$ in a neighborhood $S$, is computed as

$$
z_{\mathrm{p}}^{\prime}=\frac{\sum_{\mathrm{q} \in S} \mathrm{G}_{\sigma_{s}}(\mathrm{p}-\mathrm{q}) \mathrm{G}_{\sigma_{a}}\left(\mathbf{a}_{\mathrm{p}}-\mathbf{a}_{\mathrm{q}}\right) z_{\mathrm{q}}}{\sum_{\mathrm{q} \in S} \mathrm{G}_{\sigma_{s}}(\mathrm{p}-\mathrm{q}) \mathrm{G}_{\sigma_{a}}\left(\mathbf{a}_{\mathrm{p}}-\mathbf{a}_{\mathrm{q}}\right)} .
$$

The points $\mathrm{p}$ and $\{\mathrm{q}\}$ are the $3 \mathrm{D}$ spatial positions of corners, with corresponding attribute vector values $\mathbf{a}_{\mathrm{p}}$ and $\left\{\mathbf{a}_{\mathrm{q}}\right\}$, respectively, and $S$ specifies the spatial neighborhood around $\mathrm{p}$, corresponding to the spatial extent of the filter. The multivariate Gaussian weighting functions

$$
\mathrm{G}_{\sigma_{s}}(\mathbf{x})=e^{-\|\mathbf{x}\|^{2} / 2 \sigma_{s}^{2}}, \quad \text { and } \quad \mathrm{G}_{\sigma_{a}}(\mathbf{a})=e^{-\|\mathbf{a}\|^{2} / 2 \sigma_{a}^{2}} \text {, }
$$

determine the amount of smoothing in the spatial and attribute dimensions, respectively, via their respective parameters $\sigma_{s}$ and $\sigma_{a}$. To filter $z$ coordinates, we use Gaussian weights taking into account spatial distances as well as all attributes, i.e., $\mathbf{x} \in \mathbb{R}^{3}$, and $\mathbf{a} \in \mathbb{R}^{m}$ is a vector comprising all $m$ attribute channels. This approach smoothes small details in $z$ coordinates as shown in Fig. 5 (white dashed box), while avoiding oversmoothing $z$ values where the attributes are not homogenous (cyan dashed box).

However, for filtering attributes we use Gaussian weights based only on spatial distances and the attribute to be filtered itself, i.e., in this case we filter in $3+1$ dimensions only. The filtered attribute value $a_{\mathrm{p}}^{\prime} \in \mathbb{R}$ at position $\mathrm{p} \in \mathbb{R}^{3}$ of a corner-point, with attribute values $\left\{a_{\mathrm{q}}\right\}_{\mathrm{q} \in S}$ (all from the same channel) in a neighborhood $S$, is computed as

$$
a_{\mathrm{p}}^{\prime}=\frac{\sum_{\mathrm{q} \in S} \mathrm{G}_{\sigma_{s}}(\mathrm{p}-\mathrm{q}) \mathrm{G}_{\sigma_{a}}\left(a_{\mathrm{p}}-a_{\mathrm{q}}\right) a_{\mathrm{q}}}{\sum_{\mathrm{q} \in S} \mathrm{G}_{\sigma_{s}}(\mathrm{p}-\mathrm{q}) \mathrm{G}_{\sigma_{a}}\left(a_{\mathrm{p}}-a_{\mathrm{q}}\right)} .
$$




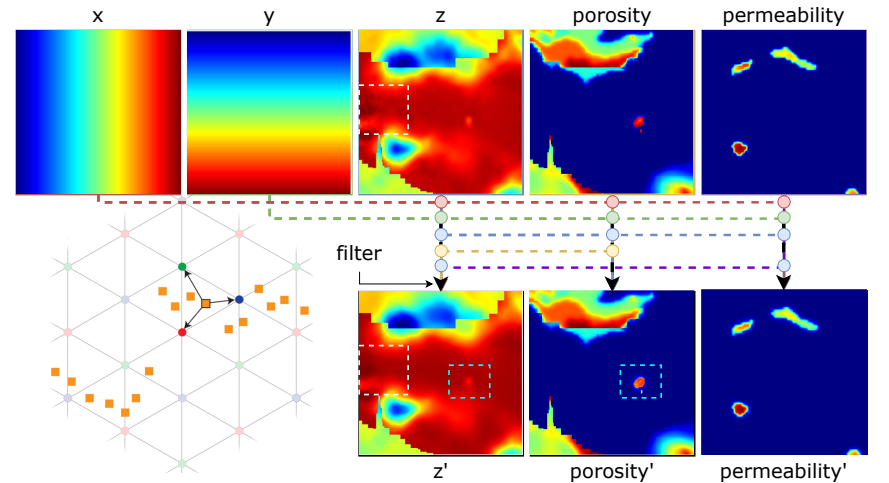

Fig. 5. Joint bilateral filtering. The $z$ coordinates are filtered using weights from the $(x, y, z)$ coordinates and all $m$ attribute channels, i.e., filtering is done in $3+m$ dimensions. Each attribute channel is filtered using weights given by $(x, y, z)$ and only that attribute, i.e., $3+1$ dimensions. Top: Input channels. Bottom right: Filtered channels. Each channel is color-coded, and dots denote channels used for filtering. Bottom left: Splatting on a 2D permutohedral grid (from [34]).

As above, the points $\mathrm{p}$ and $\{\mathrm{q}\}$ are the $3 \mathrm{D}$ spatial positions of the corners. However, now their corresponding attribute values $a_{\mathrm{p}}$ and $\left\{a_{\mathrm{q}}\right\}$ are scalars chosen from the same attribute channel.

Note that in practice, $\sigma_{s}$ and $\sigma_{a}$ are vector-valued, allowing us to control the amount of smoothing applied to each channel individually. Thus, we can smooth the different channels with different levels of smoothing depending on our needs. Conceptually, we can even set the smoothing parameter to infinity to channels that we do not want to consider in the joint smoothing. Similarly, we can easily handle boundary conditions defined by the active number attribute (binary value that indicates a cell's validity in simulation/visualization), e.g., by using $\sigma=0.01$ so that only cells whose active number is 1 will contribute to the filtering. While expert users will have some intuition of good smoothing parameters based on past trial-and-error experience, for non-experts, we recommend using a $\sigma$ parameter that is equal to the standard deviation for $z$ and attribute channels, and the average distance across 5 cells for $x, y$ channels, as a good starting point. The parameter can then be increased, e.g., doubled, for each coarser level.

\subsection{High-Dimensional Filtering w/ Permutohedral Grids}

High-dimensional Gaussian filtering is computationally expensive since an image is filtered nonlinearly with weights from a high-dimensional Gaussian function. Several approaches, e.g., the bilateral grid [36], the Gaussian KDtree [37], and filtering on a permutohedral grid [34], make this process much more efficient by reformulating the nonlinear filter as a computationally cheaper linear Gaussian convolution in a high-dimensional joint spatial and intensity domain, performing normalization afterward. In our work, we use the permutohedral grid, which is recommended by Adams et al. [34] for performing bilateral filtering in more than four dimensions, and up to 20 dimensions. In higher dimensions, the Gaussian KD-tree is more efficient. We usually filter images with at least four dimensions $((x, y, z)$ and one or more attributes, i.e., $3+m$ dimensions) making the permutohedral grid a reasonable choice.

High-dimensional Gaussian filtering using the permutohedral grid, or $p$-grid for short, is expressed in the following high-dimensional form

$$
u_{i}{ }^{\prime}=\sum_{j=1}^{n} u_{j} \cdot e^{-\frac{1}{2}\left\|\mathbf{f}_{i}-\mathbf{f}_{j}\right\|^{2}}
$$

where $n$ is the number of samples or data points. Each value $u_{i}$ to be filtered is associated with a position $\mathbf{f}_{i}$. Values with nearby positions will be maximally blurred together, while avoiding the mixing of values with non-nearby positions. In a typical color bilateral filter, the values are $u_{i}=\left[r_{i}, g_{i}, b_{i}, 1\right]$, i.e., the homogeneous color of pixel $i$. The last component of 1 leads to proper normalization by the sum of the filter weights. The positions are $\mathbf{f}_{i}=\left[\frac{r_{i}}{\sigma_{c}}, \frac{g_{i}}{\sigma_{c}}, \frac{b_{i}}{\sigma_{c}}, \frac{x_{i}}{\sigma_{s}}, \frac{y_{i}}{\sigma_{s}}\right]$, where $(x, y)$ correspond to pixel positions or indices, $\sigma_{c}$ is the color standard deviation, and $\sigma_{s}$ is the spatial standard deviation of the filter.

In our framework, we use the per-corner position $\left(p_{i}=\right.$ $\left\{x_{i}, y_{i}, z_{i}\right\}$ and attribute $\left(\mathbf{a}_{i}\right)$ channels of geometry images for filtering. To filter the $z$ coordinates, we perform $(3+m)$ dimensional filtering by defining

$$
\begin{aligned}
u_{i} & =\left[z_{i}, 1\right], \\
\mathbf{f}_{i} & =\left[\frac{x_{i}}{\sigma_{s}}, \frac{y_{i}}{\sigma_{s}}, \frac{z_{i}}{\sigma_{s}}, \frac{\mathbf{a}_{i}}{\sigma_{a}}\right], \quad(3 \text { components })
\end{aligned}
$$

For each (scalar) attribute $a$, we perform $(3+1)$-dimensional filtering by defining

$$
\begin{aligned}
& u_{i}=\left[a_{i}, 1\right], \\
& \mathbf{f}_{i}=\left[\frac{x_{i}}{\sigma_{s}}, \frac{y_{i}}{\sigma_{s}}, \frac{z_{i}}{\sigma_{s}}, \frac{a_{i}}{\sigma_{a}}\right], \quad \text { (4 components). }
\end{aligned}
$$

The $d$-dimensional permutohedral grid or p-grid is the projection of the scaled regular grid $(d+1) \mathbf{Z}^{d+1}$ along the vector $\mathbf{1}=[1, \ldots, 1]$ onto the hyperplane $H_{d}: \mathbf{x} \cdot \mathbf{1}=0$, which is the subspace of $\mathbb{R}^{d+1}$ in which coordinates sum to zero [34]. The permutohedral lattice is a tessellation of high-dimensional space that has properties that are suitable to the splat-blur-slice pipeline [34], [36], [37] for efficient bilateral filtering. This pipeline on the p-grid goes as follows. First, the position vectors are embedded in highdimensional space tessellated by the lattice. Then, each input value splats onto the vertices of its enclosing simplex using barycentric weights (Fig. 5). The enclosing simplex of any point and its barycentric coordinates can be computed efficiently in $O\left(d^{2}\right)$ time making splatting very fast. Next, lattice points blur their values with nearby lattice points using a separable Gaussian filter. Neighbors of lattice points are very efficient to compute, making the blur step highly efficient. Finally, the space is sliced at each input position using the same barycentric weights to interpolate output values. Barycentric weights that were computed from the splatting stage can be reused, thus making slicing very fast as well. We achieve between $5 \times$ to $10 \times$ speedups using the p-grid approach compared to a naive implementation of a nonlinear Gaussian filter.

\section{Encoding AND GPU Data Structure}

Our data structure compactly encodes the wavelet transform of each scale in our multiscale data representation. Smoother data regions in coarse levels have sparser wavelet transforms, thus resulting in more compact encoding, i.e., 

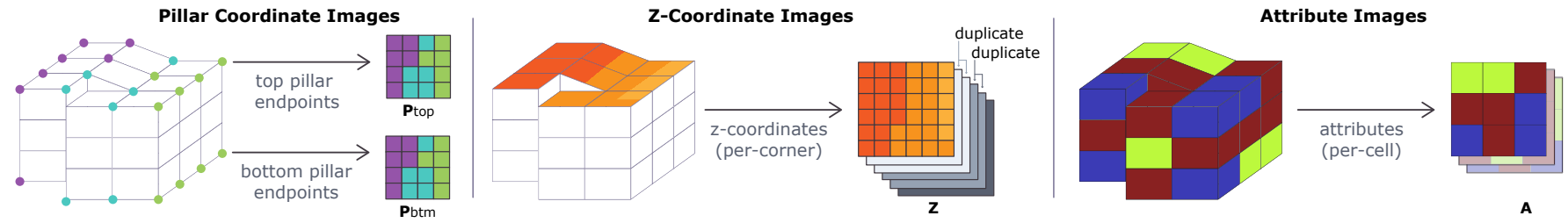

Fig. 6. Data representation. We encode a corner-point grid in image stacks comprising pillar- and $z$-coordinates as well as attribute images.

fewer bits and samples to process in coarse levels. Unlike traditional wavelet-based techniques, we use a whole wavelet transform hierarchy to represent a coarse scale instead of just a single level of approximation coefficients. This allows us to leverage wavelet-based encoding benefits, e.g., sparsity, compactness, and hierarchical structure, while, more importantly preserving level-of-detail features.

Our data structure encodes three types of images illustrated in Fig. 6: pillar coordinate images, $z$ images, and attribute images. We compress the images using our waveletbased encoding. At runtime, the $z$ and attribute images are decoded on-the-fly and on-demand while the whole pillar coordinate images are decoded once and then stored and accessed as two texture images. Note the difference in resolution of the images. Each pillar coordinate image has a resolution of $\left(C_{i}+1\right) \times\left(C_{j}+1\right)$ pixels with three channels $((x, y, z)$ of pillar endpoint). Each $z$ image has a resolution of $2 C i \times 2 C j$, i.e., the same $z$ image channel in our geometry images $I_{g}$. However, instead of $2 C_{k}$ images, we only encode $C_{k}+1$ images since we do not encode duplicate $z$ images from overlapping cell faces. (For every pair of top and bottom cells, the bottom faces of top cells are equal to the top faces of bottom cells.) Each attribute image has a resolution of $C_{i} \times C_{j}$ pixels. We encode $C_{k}$ such images. Each attribute is encoded separately from other attributes.

Our encoding, illustrated in Fig. 8, consists of two main steps, discrete wavelet transform (DWT) (Sec. 5.1) and PROGRES encoding (Sec. 5.2), resulting in our compact data structure (Sec. 5.3). During rendering, this process is reversed, i.e., PROGRES decoding followed by inverse discrete wavelet transform (IDWT).

Our wavelet-based encoding of our multiscale representation results in a compact data structure that is suitable for parallel rendering. In order to facilitate parallel processing, we subdivide the data spatially using two levels of granularity. First, we subdivide the original data in 3D spatial regions called tiles. Each tile is a user-defined 3D size, i.e., $T_{i} \times T_{j} \times T_{k}$ cells along each spatial dimension (we use $2^{2+L} \times 2^{2+L} \times 4$ where $L$ is the number of resolution levels). Then we further subdivide each tile into tilelets which are of user-defined size of $t_{i} \times t_{j} \times t_{k}$ cells along each spatial dimension (we use $8 \times 8 \times 2$ ). We perform the encoding and decoding process in tilelet granularity, i.e., tilelet images. Each compressed bytestream will correspond to compressed sub-images of $t_{i} \times t_{j}$ cell regions of tilelet images. The following therefore describes the encoding process applied on tilelet image granularity (Sec. 5.1 and 5.2) followed by the aggregation of the compressed bytestreams into a single GPU data structure (Sec. 5.3).

\subsection{Discrete Wavelet Transform}

First, we apply discrete wavelet transform (DWT) to the input tilelet image in order to get its wavelet transform coefficients. Our wavelet algorithms work on integer-valued images so we first map floating-point images to integer values using a user-defined precision (we typically map $[-1.0,1.0]$ to $\left[-10^{5}, 10^{5}\right]$ similar to HexaShrink [8]). During decoding, the integers are mapped back to floats. The DWT decomposes a tilelet image into a hierarchy of approximation and detail coefficients. The sparsity in these coefficients is leveraged by the succeeding encoding towards a compact representation. We use Haar wavelets for both $z$ and attribute data. Besides being efficient to compute, intuitively, when using Haar wavelets, smooth or homogenous regions correspond to detail coefficients that are equal to zero. This is why as we introduce more smooth data regions in coarser levels via filtering, the encoded representation also gets sparser and more compact (we apply a threshold, e.g., 0.05 , to zero out very small coefficients). We leverage this in our data structure by only encoding $\max \left(1, \frac{T_{i}}{t_{i} \cdot 2^{\eta}} \times \frac{T_{j}}{t_{j} \cdot 2^{\eta}}\right)$ tilelet images per tile image, where $\eta \in\left[0, \log _{2}\left(\max \left(\frac{T_{i}}{t_{i}}, \frac{T_{j}}{t_{j}}\right)\right)\right]$ is the finest DWT coefficient level of a tile image where not all coefficients are zerotree roots. More intuitively, when $\eta>0$, all $2^{\eta} \times 2^{\eta}$ pixel neighborhoods in a tile image are homogenous. This $\eta$ is computed hierarchically from a tile's wavelet transform coefficients. Note that enforcing $\eta=\ell$ is equivalent to using compressed mipmap images where the resolution is reduced in every level by a factor of 2 along each dimension.

\subsection{PROGRES Wavelet Transform Coding}

We encode the tilelet image's wavelet transform coefficients into a compact bytestream using a modified version of the progressive resolution coding algorithm (PROGRES) of Cho and Pearlman [31]. PROGRES, described in Algorithm 1, is a high-speed resolution-scalable wavelet transform encoding. Similar to other wavelet-based image coding methods, such as EZW [25], EBCOT [26], SPIHT [27], WDR [28], or ASWDR [29], using intra- and inter-band coding contexts, PROGRES is based on two properties of natural images: a) the energy in each subband normally decreases with spatial frequency [22], and b) statistics in a local neighborhood are similar. Thus, it also uses the strategy of coding wavelet coefficients following the order of expected importance from most to least significant bits, from low to high resolution subbands. However, unlike most coding methods that encode the ordered list of significant coefficients in bitplane-based coding, PROGRES follows a fixed Z-ordering or Morton-ordering, as depicted in Fig. 7. Therefore, it does not need to explicitly encode the ordering of significant coefficients, and each coefficient, represented by sign and 


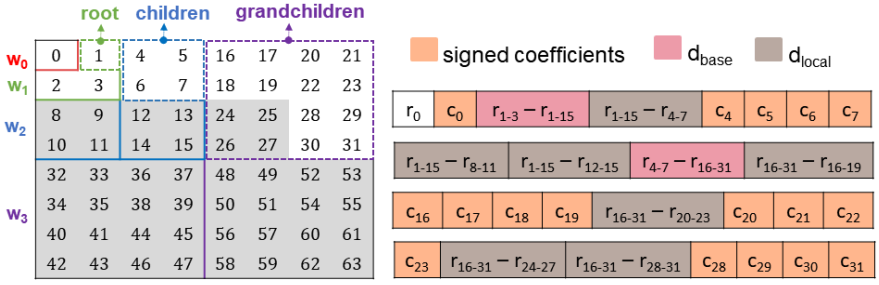

Fig. 7. PROGRES encoding uses binary coding of dynamic range and coefficient values (right) of the sparse wavelet transform image (left: zero coefficients shaded in gray). The fixed Morton scanning order (0 - 63) and example tree $t_{1}$ (dashed lines) are also shown on the left.

magnitude, is only processed once. Furthermore, PROGRES encodes each $2 \times 2$ coefficient neighborhood using the same number of bits. This number of bits as well as the sign and magnitudes of the coefficients are compactly encoded in a bytestream using dynamic range numbers described below.

We use $c_{m}$ and $t_{m}$ to represent, respectively, a wavelet coefficient at Morton index or location $m$, and a tree of coefficients with root at location $m$ (includes coefficient at $m$ together with all its children up to the finest resolution level). To illustrate this, we highlight in dashed lines the tree $t_{1}$ in Fig. 7 , i.e., tree with root at $m=1$. We encode each coefficient compactly using 1 bit for its sign, and using $r$ bits for its magnitude. The number of bits $r$ used to represent a coefficient magnitude is called the dynamic range number or dynamic range for short. Each tree will contain a different dynamic range of magnitudes, based on the activity of its coefficients. We define the dynamic range $r_{m}$ of a tree $t_{m}$ as

$$
r_{m}=\left\lceil\log _{2}\left(\max _{c_{q} \in t_{m}}\left|c_{q}\right|+1\right)\right\rceil .
$$

This accounts for how many bits are required to represent every coefficient magnitude in the tree without quantization loss. This tree-like structure of the dynamic range numbers partitions the coefficient image into a number of nonoverlapping spatial orientation trees [27], each rooted at a coefficient tree root $t_{m}$. Since the dynamic range number $r_{m}$ of tree $t_{m}$ is the maximum dynamic range for the coefficient $c_{m}$ and all its children up to the finest level, we know that if $r_{m}=0$, all the children must also have zero dynamic range, meaning their coefficients are all zeroes (zerotree with zerotree root $c_{m}$ ) and thus do not need further coding.

Using the dynamic range number information, we encode the coefficient image compactly using Algorithm 1. Before the hierarchical encoding, the first four coefficients $\left(c_{0}, c_{1}, c_{2}, c_{3}\right)$ are encoded separately first (statements 2-9). The main coding algorithm (statements 13-24) then encodes each tree of wavelet coefficients successively from low to high spatial resolution. Given $W$ resolution levels in the wavelet transform ( 0 for the lowest resolution and $W-1$ for the highest), the main coding is applied with trees rooted from resolution $w=0$ to $w=W-2$. In each encoding pass, the actual coefficients that will be encoded are the grandchildren of the current tree root. Thus, the encoding only needs to be performed for tree roots up to level $w=W-2$ (statement 11). In each pass, the Morton indices of non-zerotree roots for the next resolution level that needs to be coded will be added to the list $\mathrm{T}$. This allows zerotree skipping (statement 17) since zerotree roots are not added to T. Note that the scanning order shown in Fig. 7 results in a breadth-first-search algorithm. The coefficients in the next higher level resolution will not be processed until the ones in the current resolution level are all finished.

At its core, the algorithm encodes coefficient signs and magnitudes in batches of $2 \times 2$ neighborhoods (statement 23). Each coefficient in this batch of $2 \times 2$ coefficients is encoded using $1+r_{p}$ bits where 1 bit is used for the sign and $r_{p}$ bits are used for the magnitude. Here, $r_{p}$ is simply the maximum dynamic range number of the four coefficients in the neighborhood. For example, coefficients $c_{16}, c_{17}, c_{18}, c_{19}$ will be encoded using $r_{p}=\max \left(r_{16}, r_{17}, r_{18}, r_{19}\right)$. However, instead of directly encoding $r_{p}$ in the bytestream, PROGRES uses a two-stage differential coding of dynamic range numbers. In the first stage, $d_{\text {base }}$ or the difference between the maximum dynamic range of the children of $t$, i.e., $r_{w+1}$, and the maximum dynamic range of its grandchildren, i.e., $r_{w+2}$, is encoded (statement 15-16). Then, in the second stage, for each child $p$ of $t, d_{\text {local }}$ or the difference between $r_{w+2}$ and the maximum dynamic range of the children of $p$ (the actual $2 \times 2$ coefficient neighborhood to be encoded next) is encoded (statement 20-21). During decoding, we therefore decode $r_{w+2}$ using $r_{w+2}=r_{w+1}-d_{\text {base }}$ once for each $4 \times 4$ coefficient neighborhood (since they share the same $r_{w+2}$ ), then decode $r_{p}$ using $r_{p}=r_{w+2}-d_{\text {local }}$ for each $2 \times 2$ coefficient. Given $r_{p}$, we can then read and decode the next $4 \times\left(1+r_{p}\right)$ bits for the $2 \times 2$ coefficients.

Unary coding is used for coding the dynamic range number $r_{0}$ and the difference values $d_{\text {base }}$ and $d_{\text {local }}$. The unary code for an integer number is that number of $1 \mathrm{~s}$ followed by a single 0 . For example 1 will be encoded as 10,2 as 110,3 as 1110, and so on, while 0 is simply encoded as 0 . Unlike the original PROGRES algorithm, we allow negative values for $d_{\text {base }}$ to accommodate high detail coefficient magnitudes in $z$ images. For encoding $d_{\text {base, }}$ we therefore include an additional sign bit in the beginning where 1 is negative and 0 is positive. Therefore, +1 will be

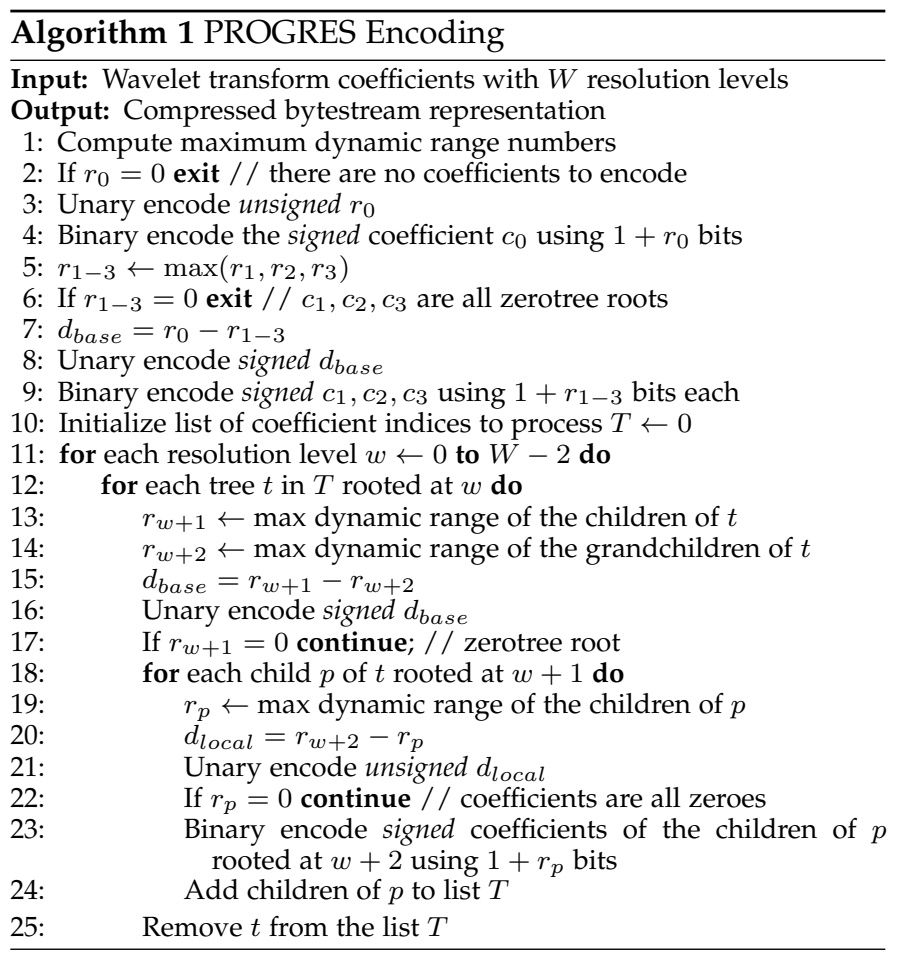


encoded as $010,-1$ as $110,+2$ as 0110 and -2 as 1110 , and so on, while we encode 0 as 00 . Fig. 7 shows the encoding of a very simple example.

PROGRES coding offers a good balance of compactness and low-complexity and memory-efficient coding which matches our requirements. Its low complexity stems from not using bit-plane coding and sophisticated entropy coding techniques such as Huffman or arithmetic coding [38]. Consequently, it does not have high memory requirements during decoding, which is crucial for our memory-limited GPU shader-based approach. More importantly, unlike the other standard methods, its compression rates do not suffer dramatically from decreasing image sizes, e.g., for small $t_{i} \times t_{j}$ tilelet images.

\subsection{Data Structure}

The resulting tilelet bytestreams for each image in our multiscale data are organized and stored in our data structure (see Fig. 8). Since our compression method is adaptive, the size of each compressed bytestream varies per tilelet image. To enable random access, we therefore store two data buffers for each data channel: the bytestream buffer $(b)$ contains compressed bytestreams concatenated and sorted in a linear fashion, and the corresponding offset buffer $(o)$ that contains the offset into the bytestream buffer for each tilelet. PROGRES decoding only needs to know the starting byte position and will terminate once decoding is finished so it does not require the length of the bytestream in advance. The offset list consists of number of tilelets per image $\times$ number of images offset values since we perform the encoding on an image basis. In addition, for each tile image, we encode $\eta$ (described in Sec. 5.1) using 8 bits. The data structure encodes the pillar coordinate images as two 2D images (top and bottom $(x, y, z)$ coordinates) using standard lossless floating-point compression. For simplicity, we use standard gzip. Recall that the pillar coordinates are decoded and loaded as GPU textures only once during initialization.

In order to reduce renderer loading times, we also precompute tile bounding boxes and store them in the data structure with additional render-time storage overhead of

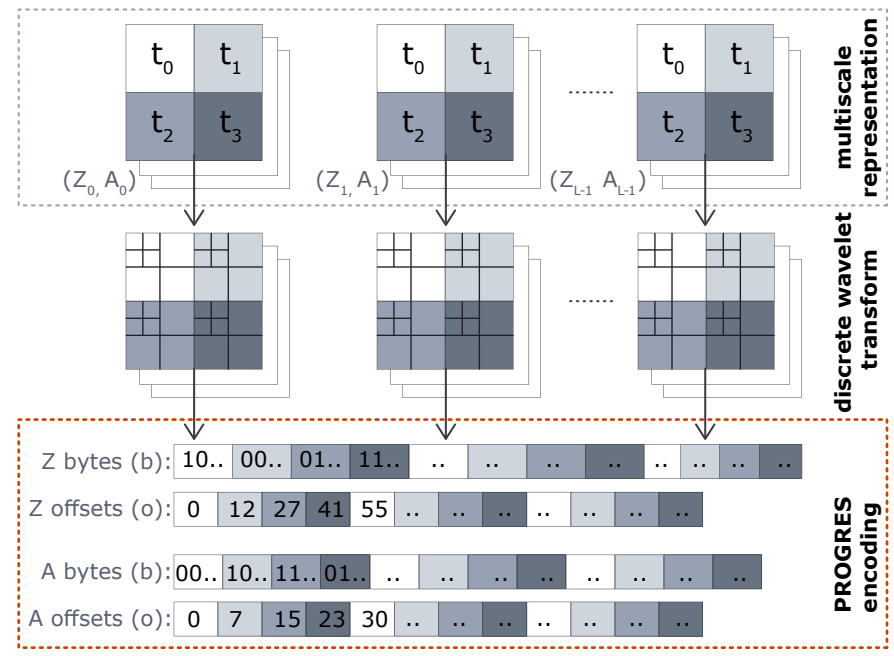

Fig. 8. Image tilelets. Our data structure (bottom, in orange dashed box) mainly comprises $z$ and attribute tilelet images, compressed into compact bytestreams (b) and a list of byte offsets (o). six float values (for minimum and maximum $(x, y, z)$ co- $^{-}$ ordinates) per tile. These bounding boxes will be used for hierarchical visibility culling during rendering.

\section{Adaptive LeVel-OF-Detail Rendering}

Each render cycle consists of three main stages as illustrated in Fig. 9. First, visible tiles and the corresponding levelof-detail at which they should be rendered is determined (Sec. 6.1). Then encoded wavelet transforms of tilelets are fetched from the data structure and decoded (Sec. 6.2). Geometric primitives, i.e., triangle vertices, indices, and attributes, are then adaptively generated on-the-fly directly from the decoded wavelet transforms (Sec. 6.3).

Unlike traditional rendering techniques that require a pre-defined vertex and index array-based geometry representation, our approach adaptively generates geometric primitives directly from our compressed representation in each rendered frame. Scalable frame-by-frame decoding and onthe-fly geometry generation is made possible using task and mesh shaders [39] that replace conventional vertex and geometry shaders in the GPU rasterization pipeline. Task and mesh shaders have very flexible compute-like capabilities for adaptive work generation in task shaders, and adaptive geometry generation in mesh shaders. Both operate in parallel thread workgroups called task workgroups and mesh workgroups in task and mesh shaders, respectively. Our mesh workgroups operate on another tiling granularity which we call a meshlet. While a mesh workgroup can typically handle up to 256 vertices (hardware-dependent), we follow the recommended maximum size of 64 vertices [39], leading to our fixed meshlet size of $2 \times 2 \times 2$ cells which we denote as $m_{i} \times m_{j} \times m_{k}$. We describe the three stages of our rendering pipeline in detail below.

\subsection{Tile Visibility and Level-of-Detail Computations}

In the first stage, a compute shader (one thread per tile) uses camera settings and tile bounding boxes to compute visibility and corresponding resolution level or level-of-detail per tile. Our visibility culling approach uses frustum culling [40] followed by hierarchical occlusion culling [41]. Our hierarchical occlusion culling uses two rendering passes. On the first pass, we render the tiles that were visible in the previous frame without using occlusion culling. We then compute the hierarchical depth map of the rendered result, i.e., an image pyramid of the depth buffer where coarse values correspond to the depth of the closest rendered object per pixel. On the second pass, we render all tiles with occlusion culling using the hierarchical depth map for occlusion computations. For each visible tile, we then use the following to determine its target resolution level: $\ell=\min \left(\left\lfloor\log _{2} \frac{B \cdot \max \left(T_{i}, T_{j}\right)}{S}\right\rfloor, L-1\right)$, where $S$ is the maximum pixel dimension of the tile's screen-space projection, and $B$ is a user-defined level-of-detail bias which we set to 1 by default. The list of visible tiles and their corresponding resolution levels serves as input to the task shader stage. Finally, the number of tilelets per tile, which corresponds to the number of task shader workgroups to deploy, is also computed using the encoded $\eta$ in the data structure (see Secs. 5.1 and 5.3) using: $\max \left(1, \frac{T_{i}}{t_{i} \cdot 2^{\eta}} \times \frac{T_{j}}{t_{j} \cdot 2^{\eta}}\right)$. 


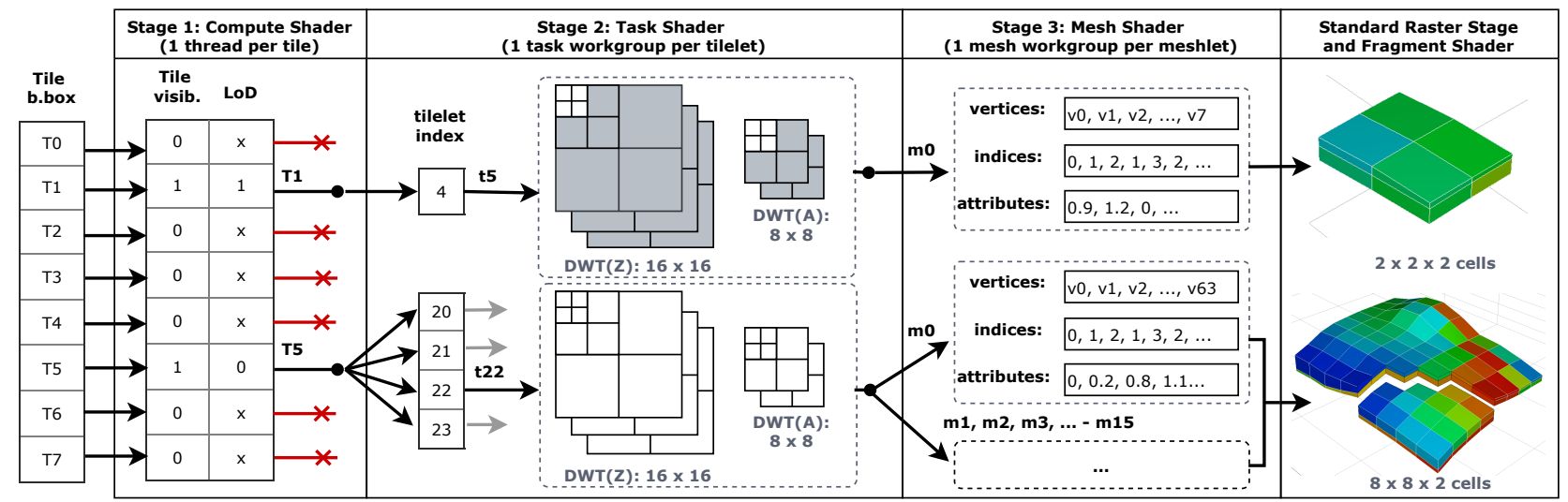

Fig. 9. Adaptive rendering overview. Stage 1: Compute shader threads perform visibility culling and level-of-detail selection for each tile. Fewer tasks are deployed for homogenous tiles (higher $\eta$ ). Stage 2: Task shader workgroups decode $z$ and attribute tilelet images. For sparse tilelet images (high $\gamma$ ), e.g., $t_{5}$ (zero coefficients shaded gray), few mesh shader workgroups are deployed $\left(m_{0}\right)$, while for non-sparse tilelet images (low $\gamma$ ), e.g., $t_{22}$, more workgroups are deployed $\left(m_{0}-m_{15}\right)$ generating finer detailed geometry. Stage 3: Mesh shader workgroups generate vertex, index, and attribute data for their assigned meshlets $(2 \times 2 \times 2$ cells $)$ that will be processed by the standard raster stage and fragment shader.

\subsection{Tilelet Decoding and Adaptive Resolution}

In the second stage, task shader workgroups (one task workgroup per tilelet) decodes tilelet bytestreams. For each tilelet, the task workgroup fetches and decodes the tilelet's bytestream for $z$ and attribute images. Each tilelet with $8 \times 8 \times 2$ cells requires three image slices of $z$ values (top, middle, bottom faces) and two slices of attribute values (top, bottom cells) as shown in Fig. 9 (Stage 2). The task workgroup then dispatches mesh workgroups that will eventually generate geometry using the decoded images. The offset of each tilelet's bytestream is fetched from the offset data in the data structure. The PROGRES decoding is performed in parallel, i.e., one thread decodes one tilelet image's wavelet transform coefficients. This is followed by a parallel IDWT which recovers the encoded tilelet images. The decoded images are written in a memory block that is accessible to mesh workgroups that will be dispatched by the task shader workgroup. Since the task shader output memory block has a hardware-dependent limit, we have to select the tilelet size such that the total size of the $z$ and attribute images fits within this limit. For instance, for the typical limit of $16 \mathrm{~KB}$, we can set each tilelet to have up to $16 \times 16 \times 2$ cells resulting in $3(32 \times 32)$-float and $2(16 \times 16)$ float images for $\mathrm{z}$ and attributes, respectively, for a total of 14,336 Bytes. In practice, however, we use tilelets with $8 \times 8 \times 2$ cells $(3,584$ Bytes) to maximize GPU occupancy and increase frame rates.

Our wavelet-based encoding enables our adaptive mesh rendering approach to generate and render fewer triangles for smooth data regions compared to high-detail regions, similar to adaptive mesh rendering approaches [42], [43]. On the tilelet level, this adaptiveness is achieved by generating few primitives for zerotree coefficients [25] in the tilelet's wavelet transform since they correspond to homogenous regions. More specifically, the number of mesh workgroups $m$ that a task shader dispatches is computed using $m=$ $\max \left(1, \frac{t_{i}}{m_{i} \cdot 2^{\gamma}} \times \frac{t_{j}}{m_{j} \cdot 2^{\gamma}}\right)$ where $\left(m_{i}, m_{j}\right)$ are our meshlet sizes along $x, y$, respectively, and $\gamma \in\left[0, \log _{2}\left(\max \left(\frac{t_{i}}{m_{i}}, \frac{t_{j}}{m_{j}}\right)\right)\right]$ is the finest DWT coefficient level of a tilelet image where not all coefficients are zerotree roots. This $m$ is computed hierarchically from the tilelet's wavelet transform during the task shader stage. Overall, more zerotrees in DWT coefficients lead to higher $\eta$ and $\gamma$, resulting in fewer task and mesh work to dispatch and thus fewer geometry to generate and rasterize. Together, $\eta$ and $\gamma$ computed from our wavelet encoding ultimately enables adaptive rendering. Consequently, the per-cell footprint is equivalent to $2^{\eta} \times 2^{\gamma}$ cells along $x$ and $y$. We use the minimum $\eta$ and $\gamma$ considering both the $z$ and currently active attribute coefficients in order to display fine details in both the mesh and attribute.

\subsection{Geometry Generation}

In the third stage, mesh shader workgroups (one mesh workgroup per meshlet) generate the vertex and index lists as well as per-vertex and per-triangle attributes of meshlets. Mesh workgroups also perform attribute-based visibility culling, i.e., generating geometry only for cells whose attributes fall within a user-defined range, which is an important feature for geological model analysis. The same mechanism is used to skip rendering of non-active cells. Each mesh shader workgroup generates geometry for at most $2 \times 2 \times 2=8$ cells. Given $\eta$ and $\gamma$ passed from the parent task workgroup, a mesh workgroup adjusts the spatial footprint of each of its cells to cover $2^{\eta} \times 2^{\gamma}$ cells along $x$ and $y$. Each mesh workgroup fetches the $z$ coordinates of the cells corresponding to its designated region-of-interest from the $z$ images decoded by its parent task workgroup. The $(x, y)$ coordinates can be computed by linear interpolation using the decompressed $(x, y, z)$ coordinates of the top and bottom pillar textures. Once the $x, y, z$ coordinates of the cells have been computed, the vertex and index lists are generated based on the implicit hexahedral cell topology (see Fig. 3). A similar process is applied to the attribute data. The mesh workgroup then generates per-vertex attribute scalars and per-primitive normals for shading. Mesh shader output is then processed by the standard raster stage and fragment shader resulting in the final rendered image.

\section{Results}

We demonstrate results using real-world geological models as well as synthetic models generated using the Matlab Reservoir Simulation Toolbox (MRST) [9]. Synthetic models are often used in place of proprietary and confidential realworld data for research and development purposes. The 

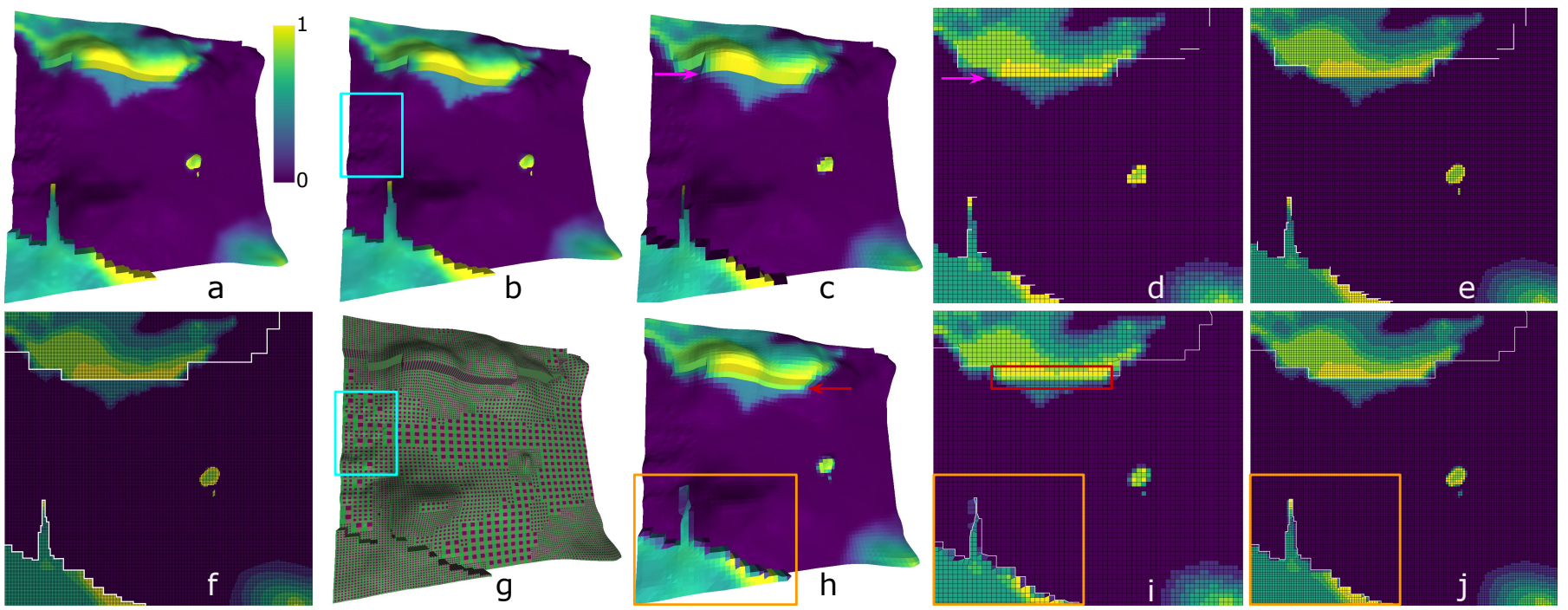

Fig. 10. Preserving features. Original data: 3D visualization (a) and faults (white outlines) overlayed on porosity attribute of top slice (f). Our featurepreserving result (b) is achieved by adaptive rendering (each color block is a meshlet with 96 triangles) (g). Coarse resolution levels computed using subsampling: level 2 (c,d), level 1 (e) can suffer from fast degradation and loss of small details and faults (magenta arrows: faults disappear and turn into highly slanted cells). HexaShrink: level 2 (h,i), level 1 (j) is able to maintain faults but attribute edges are oversmoothed (red arrow and box: high porosity values bleeding out) and the mesh and attribute spatial positions are misaligned, i.e., drift away from each other (see regions in orange boxes). Note that our adaptive rendering considers the mesh and attribute features jointly so that even regions with homogenous or flat attributes but with non-homogenous mesh (or other way around) will be rendered with adaptively fine-detailed geometry (see region in cyan boxes).

attributes in our synthetic models (Models 2 and 3) were derived from the porosity and permeability attributes of the SPE10 dataset [44] accessible in MRST. The San Joaquin Basin (SJB) dataset is a real-world simulation of the region's geological state 22 million years prior to the present day model [45]. Table 1 lists down the datasets we used with their storage sizes in terms of the standard corner-point grid format described in Sec. 3.2.

We performed the rendering experiments on three setups: S1, S2, and S3. S1 and S2 used the same desktop PC with two $2.3 \mathrm{GHz}$ Intel Xeon E5 processors and $128 \mathrm{~GB}$ RAM. S1 used a Titan RTX GPU with 24 GB of memory, while S2 used an RTX 2080 GPU with 8 GB of memory. S3 is a laptop with an Intel Core i7 processor, 16 GB RAM, and an RTX 2070 Max-Q GPU with 8 GB of memory. Note that with the same renderer settings, all setups will render the same image at different frame rates. Our renderer is implemented in C++ and uses the Vulkan 1.2 graphics API with NVIDIA's VK_NV_mesh_shader extension [46]. Parallel preprocessing on S1 using Matlab with MRST scales linearly with an average processing time of around 5 minutes per brick of $256 \times 256 \times 64$ cells ( 32 ghost cells along $x, y$ boundaries are added during filtering to avoid inter-brick artifacts).

Below, we discuss how our approach compares with others in terms of compression, quality (feature-preservation), GPU memory footprint, and frame rates.

TABLE 1

Datasets. Synthetic data $\left({ }^{*}\right)$. \# of attributes $(m)$. Size is storage size.

\begin{tabular}{|l|l|l|l|l|}
\hline Dataset & Cell Dimensions & \# Cells & $m$ & Size (MB) \\
\hline Johansen & $144 \times 176 \times 16$ & 405,504 & 3 & 18.46 \\
\hline VK & $176 \times 80 \times 68$ & 957,440 & 3 & 42.47 \\
\hline Model 1 & $512 \times 512 \times 64$ & $16,777,216$ & 2 & 413.16 \\
\hline SJB & $2304 \times 3072 \times 32$ & $226,492,416$ & 3 & $10,135.66$ \\
\hline Model 2* & $2048 \times 2048 \times 64$ & $268,435,456$ & 2 & $10,838.18$ \\
\hline Model 3 $^{*}$ & $4096 \times 4096 \times 64$ & $1,073,741,824$ & 1 & $39,057.56$ \\
\hline
\end{tabular}

Compression. Besides enabling interactive visualization, our compressed representation can be beneficial for data storage and transmission of very large datasets. Our representation leads to compression rates that are comparable to standard methods in compressing the original data $(\ell=0)$ with minimal information loss. For a fair comparison, the encoding process was exactly the same for all methods (duplicate $z$ images were skipped, compression was performed on tilelets) except that PROGRES was replaced with GZIP, STW [47], and SPIHT [27]. The other methods can be very compact for relatively smooth data but are not necessarily directly useful for real-time GPU-based visualization.

TABLE 2

Compression ratios using our approach compared to other methods.

\begin{tabular}{|l|l|l|l|l|}
\hline Dataset & GZIP & STW & SPIHT & Ours \\
\hline Johansen & 3.13 & 3.01 & 3.77 & 4.69 \\
\hline VK & 4.07 & 4.52 & 5.54 & 8.86 \\
\hline Model 1 & 3.69 & 4.08 & 4.96 & 7.45 \\
\hline SJB & 4.20 & 3.98 & 4.57 & 12.41 \\
\hline Model 2* $^{*} 2.03$ & 3.82 & 4.98 & 7.17 \\
\hline Model 3 $^{*}$ & 2.32 & 4.89 & 6.29 & 6.86 \\
\hline
\end{tabular}

Level-of-Detail Feature Preservation. Our qualitative results demonstrate the preservation of important features across resolution levels, due to our nonlinear filtering and adaptive resolution. Fig. 10 shows a comparison of our adaptive, feature-preserving approach with standard techniques. Subsampling can lead to aliasing which means important edges on the mesh can be skipped, as we can see from the disappearing white outlines in Fig. $10(\mathrm{~d}, \mathrm{e})$, which correspond to faults. When this happens, faults disappear and become non-faulted cells that are highly slanted as highlighted by the arrows in Fig. 10 (c,d). Our approach is able to maintain faults by avoiding the oversmoothing of $z$ images via our nonlinear joint bilateral filter and adaptive mesh rendering. HexaShrink [8] is able to better maintain faults via adaptive subsampling of the mesh as we can see from the white outlines in Fig. $10(\mathrm{i}, \mathrm{j})$ that are more 
TABLE 3

GPU runtime sizes (MB) and frame rates (FPS). $L$ is \# of resolution levels. \# Triangles is for $l_{0} . \mathrm{X}=$ out of GPU memory. NS = not supported.

\begin{tabular}{|c|c|c|c|c|c|c|c|c|c|c|c|c|c|c|c|c|}
\hline \multirow{2}{*}{ Dataset } & \multirow{2}{*}{$L$} & \multirow{2}{*}{$\begin{array}{l}\text { \# Triangles } \\
\text { (Millions) }\end{array}$} & \multicolumn{2}{|c|}{ ResInsight } & \multirow{2}{*}{$\begin{array}{c}\text { Standard } \\
(\mathrm{MB})\end{array}$} & \multicolumn{3}{|c|}{ Standard (FPS) } & \multirow{2}{*}{$\begin{array}{l}\text { Task/Mesh } \\
\text { (MB) }\end{array}$} & \multicolumn{3}{|c|}{ Task/Mesh (FPS) } & \multirow{2}{*}{$\begin{array}{l}\text { Our } \\
\text { (MB) }\end{array}$} & \multicolumn{3}{|c|}{ Our (FPS) } \\
\hline & & & $(\mathrm{MB})$ & (FPS) & & S1 & S2 & S3 & & S1 & $\mathrm{S} 2$ & S3 & & S1 & S2 & S3 \\
\hline Johansen & 2 & 4.87 & 454 & 425 & 52.92 & 703 & 874 & 398 & 22.93 & 822 & 992 & 393 & 4.68 & 513 & 554 & 212 \\
\hline VK & 2 & & 734 & 286 & & 677 & 793 & 381 & & 749 & 795 & 376 & 5.47 & 340 & 356 & 161 \\
\hline Model $1^{*}$ & 3 & 201.33 & 10,314 & 184 & $2,291.11$ & 367 & 340 & 203 & 887.22 & 424 & 403 & 219 & 86.29 & 142 & 139 & 65 \\
\hline SJB & 4 & $2,717.91$ & $\mathrm{X}$ & $\mathrm{X}$ & $32,500.29$ & $\mathrm{X}$ & $\mathrm{X}$ & $\mathrm{X}$ & $13,405.73$ & NS & $\mathrm{X}$ & $\mathrm{X}$ & $1,816.60$ & 68 & 60 & 24 \\
\hline & 4 & & NS & NS & & $\mathrm{X}$ & $\mathrm{X}$ & $\mathrm{X}$ & & NS & $\mathrm{X}$ & $\mathrm{X}$ & & 66 & 57 & 23 \\
\hline Model 3* & 5 & $12,884.90$ & NS & NS & $148,791.23$ & $\bar{X}$ & $\mathrm{X}$ & $\mathrm{X}$ & $57,625.74$ & $X$ & $\bar{X}$ & $\bar{X}$ & $5,862.64$ & 34 & 25 & 12 \\
\hline
\end{tabular}

consistent with those in Fig. 10 (f). However, attributes can be oversmoothed by the LTI filter effect when using approximation coefficients of attribute wavelet transforms as their low-resolution versions. This leads to bleeding out of the high-porosity values in the high-elevation region into the low-elevation region as pointed by the red arrow and box in Fig. 10 (h,i). Furthermore, the adaptive mesh subsampling can pick mesh regions that are not aligned with the central attribute locations, which can lead to mesh and attribute misalignment as we can see in the regions inside orange boxes in Fig. $10(\mathrm{~h}, \mathrm{i}, \mathrm{j})$. Our approach is able to avoid this artifact since the resolutions of the mesh and attributes are jointly adapted, i.e., only regions where the mesh and attributes are both smooth are represented with fewer geometry and are always centrally aligned.

Fig. 11 shows that our nonlinear filtering approach is able to reduce small details while preserving main faults. In this example, the active number attribute was used in filtering with $\sigma=0.01$ to avoid the introduction of boundary artifacts as we described in Sec. 4.1. The same filtering approach was used for the VK dataset in Fig. 12.

Scalable and Interactive Visualization. Due to the compactness of our data structure, we are able to squeeze in larger data sizes in the GPU compared to other approaches while still enabling interactive frame rates. Table 3 compares our approach with other standard methods in terms of runtime sizes, i.e. GPU memory required to render the data, in megabytes (MB) and frame rates in frames per second (FPS). For reference, it also provides the total number of resolution levels, $L$, we used in the experiments and the number of triangles in $l_{0}$. ResInsight [48] is an open-source 3D visualization tool for reservoir models. ResInsight is not able to load Models 2 and 3 since they are represented as multiple GRDECL files (this is denoted as NS or not supported in Table 3). Note that ResInsight frame rates were only measured on S1 and runtime sizes are based on memory use reported in its GUI. The standard approach utilizes a pre-defined and structured vertex and index list-based representation of the data in the standard render pipeline with a generic vertex shader. Here, each cell is represented by its 8 corner vertices with 3 values each $(x, y, z$ positions) and its $m$ floating
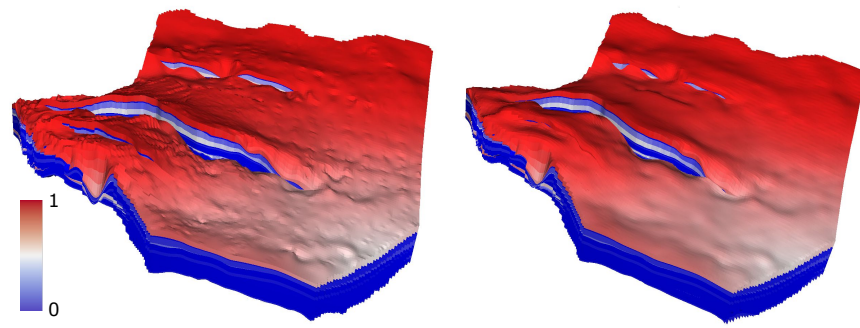

Fig. 11. Preserving faults. Left: Original Johansen data. Right: Small details are smoothed but faults are maintained after applying our filter.
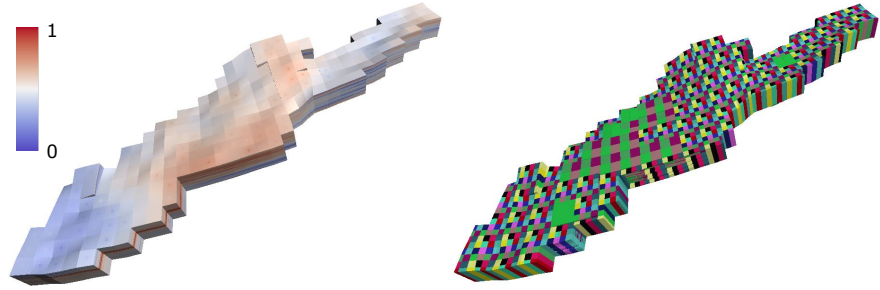

Fig. 12. VK dataset rendering (left) and meshlet visualization (right).

point attribute values. Its runtime size is roughly equal to $\left(C_{i} \cdot C_{j} \cdot C_{k}\right) \times(8 \cdot 3+m) \times 4$ Bytes $\times H$, where $H \approx 1.33$ accounts for the multiresolution hierarchy. We leverage the implicit hexahedron topology and reuse a single index list (with only 36 unsigned integer values for 12 triangles of a hexahedron) for all cells. The task/mesh approach uses the same task- and mesh-shader based rendering approach as ours without the PROGRES compression and adaptive rendering. This approach always downsamples the data along $x$ and $y$ by a factor of 2 in each resolution level and thus suffers from the quality degradation explained above. Its runtime size is based on a multiresolution version of the
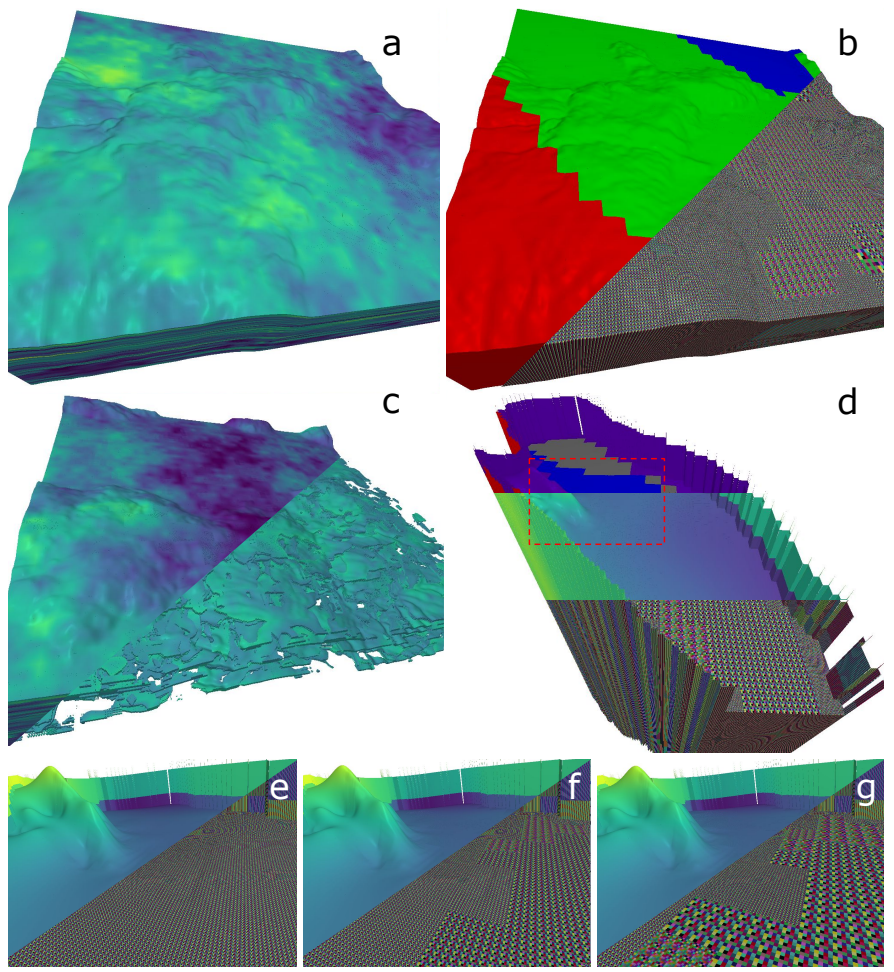

Fig. 13. Scalable visualization. Model 2 porosity visualization (a) and per-tile level-of-detail (b:top left, $l_{0}$ is red, $l_{1}$ is green, $l_{2}$ is blue) and meshlet visualization (b:bottom right). Model 3 porosity visualization (c) with filtering to show porosity within $[0.4,0.6]$ range (c:bottom right). SJB level-of-detail (d:top), pressure visualization (d:middle), with meshlet visualization (d:bottom). SJB region (d:red box) rendered at fixed resolution levels (e: $\left.l_{0}, f: l_{1}, g: l_{2}\right)$ show feature-preserving level-of-detail. 
corner-point grid format and is roughly equal to the storage size of the data in Table 1 multiplied by $H$. Our approach directly utilizes our compressed data representation with on-the-fly decoding and adaptive rendering via task and mesh shaders. The standard and task/mesh approaches use the same occlusion and frustum culling as well as indirect draws setup as our approach. In order to mimic real-world interaction when measuring frame rates, we automatically rotated and zoomed-in/out the camera and report the average FPS (for a 1,024 × 1,024 window).

Depending on the smoothness and feature content of the data, the adaptiveness of our approach will typically render more triangles than the standard and task/mesh approaches in order to better preserve features. As a tradeoff with better quality, our approach will tend to be slightly slower, especially for smaller datasets due to the decoding overhead. However, as we can see in Table 3, with SJB and Models 2 and 3 (Fig. 13), our approach can load more data locally in fast GPU memory due to its compact data representation. The standard and task/mesh approaches will fail to handle large data sets without using more complex out-of-core approaches. Note, however, that the task/mesh approach requires much less memory than the standard approach since it is able to leverage the compact pillar grid representation of the mesh. In S1, even with 24 GB of GPU memory, the task/mesh method can not directly support SJB and Model 2 due to the 4 GB limit of GPU storage buffers (thus we report NS or not supported). Overall, the three methods have different tradeoffs in terms of quality, memory footprint, and frame rates, with our approach having the best balance with the most advantage in memory requirements and quality. In terms of GPU performance comparison, the frame rates of S3 are around half of S1 across all techniques, which is consistent with the GPU specifications (S1 has 4,608 CUDA cores while S3 has $2,304)$. Our approach is able to provide acceptable frame rates even in laptop setups and commodity GPUs. Finally, as described in Sec. 6.3, our approach can support attributebased filtering as shown in Fig. 13 (c), which is important in analysis.

\section{CONCLUSION AND FutURE WORK}

We have presented a scalable multiresolution framework that results in compact representations of geological models with preserved level-of-detail features in interactive visualization. Our multiscale representation uses a nonlinear feature-preserving smoothing filter applied jointly to the mesh and attribute data in our image-based representation. Our hierarchical wavelet-based encoding and data structure exploits the sparsity in the data to reduce storage requirements and facilitate adaptive rendering. Finally, our adaptive mesh rendering leverages GPU task and mesh shaders in interactive visualization. For future work, we are interested in exploring (1) semi-automatic ways to find good filtering parameters $(\Omega),(2)$ the representation of more complex unstructured grids, (3) visualization and analytics of time-varying meshes and attributes, and (4) scaling to trillion-cell datasets via a distributed data structure and rendering approach. We hope that our work can benefit those who are working towards better understanding geological models and ultimately Earth's complex geological systems.
ACKNOWLEDGMENTS

The authors wish to thank Dr. Hussein Hoteit for providing the VK dataset. This work was supported in part by a grant from Saudi Aramco (\#3879).

\section{RefERENCES}

[1] E. LaMar, B. Hamann, and K. I. Joy, "Multiresolution techniques for interactive texture-based volume visualization," in Proceedings Visualization '99 (Cat. No.99CB37067), Oct 1999, pp. 355-543.

[2] K. Zimmermann, R. Westermann, T. Ertl, C. Hansen, and M. Weiler, "Level-of-detail volume rendering via 3D textures," in 2000 IEEE Symposium on Volume Visualization, Oct 2000, pp. 7-13.

[3] X. Li and H.-W. Shen, "Time-critical multiresolution volume rendering using 3d texture mapping hardware," in Symposium on Volume Visualization and Graphics, 2002. Proceedings. IEEE/ACM SIGGRAPH. IEEE, 2002, pp. 29-36.

[4] J. Beyer, M. Hadwiger, T. Möller, and L. Fritz, "Smooth mixedresolution gpu volume rendering," in IEEE/EG Symposium on Volume and Point-Based Graphics 2008, August 2008.

[5] R. Sicat, J. Krüger, T. Möller, and M. Hadwiger, "Sparse PDF volumes for consistent multi-resolution volume rendering," IEEE Transactions on Visualization and Computer Graphics, vol. 20, no. 12, pp. 2417-2426, 2014.

[6] H. Younesy, T. Möller, and H. Carr, "Improving the quality of multi-resolution volume rendering," in Proceedings of the Eighth Joint Eurographics / IEEE VGTC Conference on Visualization. Eurographics Association, 2006, p. 251-258.

[7] M. Hadwiger, R. Sicat, J. Beyer, J. Krüger, and T. Möller, "Sparse pdf maps for non-linear multi-resolution image operations," $A C M$ Transactions on Graphics, vol. 31, no. 6, Nov. 2012.

[8] J. L. Peyrot, L. Duval, F. Payan, L. Bouard, L. Chizat, S. Schneider, and M. Antonini, "HexaShrink, an exact scalable framework for hexahedral meshes with attributes and discontinuities: multiresolution rendering and storage of geoscience models," Computational Geosciences, vol. 23, no. 4, pp. 723-743, 2019.

[9] K. Lie, An Introduction to Reservoir Simulation Using MATLAB/GNU Octave: User Guide for the MATLAB Reservoir Simulation Toolbox (MRST). Cambridge University Press, 2019.

[10] X. Li, X. Li, and D. Zhang, "Generalized prism grid: a pillar-based unstructured grid for simulation of reservoirs with complicated geological geometries," Computational Geosciences, vol. 22, no. 6, pp. 1561-1581, Dec 2018.

[11] M. Hadwiger, J. Beyer, W. Jeong, and H. Pfister, "Interactive volume exploration of petascale microscopy data streams using a visualization-driven virtual memory approach," IEEE Transactions on Visualization and Computer Graphics, vol. 18, no. 12, pp. 22852294, Dec 2012.

[12] T. Fogal, A. Schiewe, and J. Krüger, "An analysis of scalable gpubased ray-guided volume rendering," in 2013 IEEE Symposium on Large-Scale Data Analysis and Visualization, Oct 2013, pp. 43-51.

[13] R. Westermann, "A multiresolution framework for volume rendering," in Proceedings of the 1994 Symposium on Volume Visualization. Association for Computing Machinery, 1994, p. 51-58.

[14] D. Thompson, J. A. Levine, J. C. Bennett, P.-T. Bremer, A. Gyulassy, V. Pascucci, and P. P. Pébay, "Analysis of large-scale scalar data using hixels," in 2011 IEEE Symposium on Large Data Analysis and Visualization. IEEE, 2011, pp. 23-30.

[15] S. Liu, J. A. Levine, P. Bremer, and V. Pascucci, "Gaussian mixture model based volume visualization," in IEEE Symposium on Large Data Analysis and Visualization (LDAV), Oct 2012, pp. 73-77.

[16] H. Hoppe, T. DeRose, T. Duchamp, J. McDonald, and W. Stuetzle, "Mesh optimization," in Proceedings of the 20th Annual Conference on Computer Graphics and Interactive Techniques, 1993, pp. 19-26.

[17] H. Hoppe, "Progressive meshes," in 23rd Annual Conference on Computer Graphics and Interactive Techniques, 1996, pp. 99-108.

[18] R. Pajarola and J. Rossignac, "Compressed progressive meshes," IEEE Transactions on Visualization and Computer Graphics, vol. 6, no. 1, pp. 79-93, Jan 2000.

[19] P. Cignoni, C. Montani, C. Rocchini, R. Scopigno, and M. Tarini, "Preserving attribute values on simplified meshes by resampling detail textures," The Visual Computer, vol. 15, no. 10, pp. 519-539, 1999.

[20] H. Hoppe, "New quadric metric for simplifying meshes with appearance attributes," in Proceedings of the 10th IEEE Visualization 1999 Conference (VIS '99). IEEE Computer Society, 1999. 
[21] F. Abraham and W. Celes, "Multiresolution visualization of massive black oil reservoir models," The Visual Computer, vol. 35, no. 6-8, p. 837-848, 2019.

[22] S. G. Mallat, "A theory for multiresolution signal decomposition: the wavelet representation," IEEE Transactions on Pattern Analysis and Machine Intelligence, vol. 11, no. 7, pp. 674-693, July 1989.

[23] M. Gross, L. Lippert, R. Dittrich, and S. Häring, “Two methods for wavelet-based volume rendering," Computers and Graphics, vol. 21, no. 2, pp. $237-252,1997$.

[24] T. Kim and Y. Shin, "An efficient wavelet-based compression method for volume rendering," in Seventh Pacific Conference on Computer Graphics and Applications, Oct 1999, pp. 147-156.

[25] J. M. Shapiro, "Embedded image coding using zerotrees of wavelet coefficients," Fundamental Papers in Wavelet Theory, vol. 41, no. 92, pp. 861-878, 2009.

[26] D. Taubman, "High performance scalable image compression with EBCOT," IEEE Transactions on Image Processing, vol. 9, no. 7, pp. 1158-1170, 2000.

[27] A. Said and W. A. Pearlman, "A new, fast, and efficient image codec based on set partitioning in hierarchical trees," IEEE Transactions on Circuits and Systems for Video Technology, vol. 6, no. 3, pp. 243-250, 1996.

[28] J. Tian and R. O. Wells, Embedded Image Coding Using Wavelet Difference Reduction. Springer US, 1998, pp. 289-301.

[29] J. S. Walker, "Lossy image codec based on adaptively scanned wavelet difference reduction," Optical Engineering, vol. 39, no. 7, pp. $1891-1897,2000$.

[30] J. S. Walker and T. Q. Nguyen, "Wavelet-based image compression," Sub-chapter of CRC Press book: Transforms and Data Compression, 2001.

[31] Y. Cho and W. A. Pearlman, "Hierarchical dynamic range coding of wavelet subbands for fast and efficient image decompression," IEEE Transactions on Image Processing, vol. 16, no. 8, pp. 2005-2015, Aug 2007.

[32] X. Gu, S. J. Gortler, and H. Hoppe, "Geometry images," ACM Transactions on Graphics, vol. 21, no. 3, p. 355-361, 2002.

[33] Z. Farbman, R. Fattal, D. Lischinski, and R. Szeliski, "Edgepreserving decompositions for multi-scale tone and detail manipulation," ACM Transactions on Graphics, vol. 27, no. 3, pp. 1-10, 2008.

[34] A. Adams, J. Baek, and M. A. Davis, "Fast high-dimensional filtering using the permutohedral lattice," Computer Graphics Forum, vol. 29, no. 2, pp. 753-762, 2010.

[35] L. Caraffa, J. Tarel, and P. Charbonnier, "The guided bilateral filter: When the joint/cross bilateral filter becomes robust," IEEE Transactions on Image Processing, vol. 24, no. 4, pp. 1199-1208, 2015.

[36] J. Chen, S. Paris, and F. Durand, "Real-time edge-aware image processing with the bilateral grid," ACM Transactions on Graphics, vol. 26, no. 3, pp. 103-es, 2007.

[37] A. Adams, N. Gelfand, J. Dolson, and M. Levoy, "Gaussian kdtrees for fast high-dimensional filtering," ACM Transactions on Graphics, vol. 28, no. 3, Jul. 2009.

[38] I. H. Witten, R. M. Neal, and J. G. Cleary, "Arithmetic coding for data compression," Communications of the ACM, vol. 30, no. 6, p. 520-540, 1987.

[39] C. Kubisch, "Introduction to Turing mesh shaders," last accessed February 17, 2020. [Online]. Available: https://devblogs.nvidia. $\mathrm{com} /$ introduction-turing-mesh-shaders

[40] G. Gribb and K. Hartmann, "Fast extraction of viewing frustum planes from the world-view-projection matrix," last accessed Feb. 17, 2020. [Online]. Available: https://www.gamedevs.org

[41] H. Zhang, D. Manocha, T. Hudson, and K. E. Hoff, "Visibility culling using hierarchical occlusion maps," in 24th Annual Conference on Computer Graphics and Interactive Techniques. ACM Press/Addison-Wesley Publishing Co., 1997, p. 77-88.

[42] J. C. Xia, J. El-Sana, and A. Varshney, "Adaptive real-time levelof-detail based rendering for polygonal models," IEEE Transactions on Visualization and Computer Graphics, vol. 3, no. 2, pp. 171-183, April 1997.

[43] J. K. Kim and J. B. Ra, "A real-time terrain visualization algorithm using wavelet-based compression," The Visual Computer: International Journal of Computer Graphics, vol. 20, no. 2, pp. 67-85, 2004.

[44] J. E. Aarnes, V. Kippe, and K.-A. Lie, "Mixed multiscale finite elements and streamline methods for reservoir simulation of large geomodels," Advances in Water Resources, vol. 28, no. 3, pp. 257 271, 2005.
[45] A. H. Scheirer, "Petroleum systems and geologic assessment of oil and gas in the San Joaquin Basin Province, California: U.S. Geological Survey Professional Paper 1713," last accessed July 7, 2021. [Online]. Available: http://pubs.usgs.gov/pp/pp1713/

[46] P. Brown, J. Bolz, D. Koch, P. Daniell, and P. Boudier, "Vulkan specification: VK_NV_mesh_shader," last accessed August 12, 2020. [Online]. Available: https://www.khronos.org/registry/vulkan/ specs/1.1-extensions/html/vkspec.html\#VK_NV_mesh_shader

[47] A. Said and W. A. Pearlman, "Image compression using the spatial-orientation tree," in IEEE International Symposium on Circuits and Systems, 1993, pp. 279-282.

[48] Ceetron and Equinor, "ResInsight," last accessed October 22, 2020. [Online]. Available: https://resinsight.org

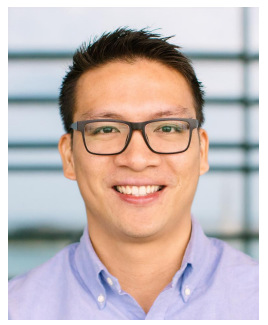

Ronell Sicat received his MSc in electrical engineering in 2010 from KAUST, Saudi Arabia. He received his $\mathrm{PhD}$ in computer science in 2015 from KAUST, supervised by Prof. Markus Hadwiger. He was a postdoctoral researcher at Harvard University from 2016 to 2018 working with Prof. Hanspeter Pfister. He is currently a research scientist in KAUST interested in immersive analytics, large-scale interactive data visualization, and multiresolution techniques.

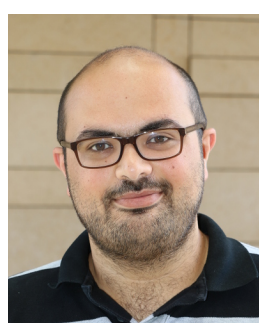

Mohamed Ibrahim earned his PhD in 2019 from King Abdullah University of Science and Technology (KAUST) focused on scientific data visualization. He then worked as a postdoctoral fellow in the High-Performance Visualization Group in KAUST where he worked on visualization of large-scale particle data and hexahedral meshes. His research interests include large-scale data visualization, ray tracing, and physically based rendering.

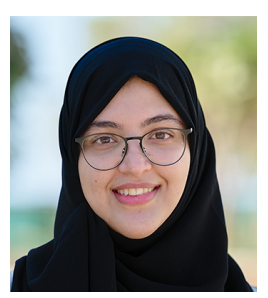

Amani Ageeli received her MSc degree in computer science from King Abdullah University of Science and Technology (KAUST), Saudi Arabia, in 2019. She is currently pursuing her computer science PhD in KAUST supervised by Prof. Markus Hadwiger. Her current research focuses on interactive large-scale visualization of scientific data, and modern GPU algorithms and architecture.

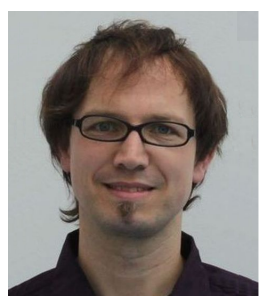

Florian Mannuss is currently a petroleum engineer systems analyst in the Strategic Modeling Technology Team in Saudi Aramco. His research interests are in large-scale simulation of basin and reservoir data in the oil and gas industry, as well as interactive visualization and analysis of such data. He is also interested in novel immersive analytics with work in augmented and virtual reality systems and environments.

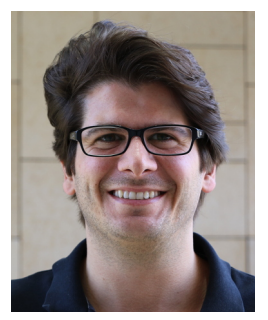

Peter Rautek received his MSc and PhD in computer science from Vienna University of Technology in 2005 and 2009, respectively. He worked as a research assistant and postdoctoral fellow at the same university between 2005 and 2011. Since 2011 until present, he is a research scientist at KAUST, Saudi Arabia, focused on interactive large-scale data visualization, virtual reality, domain-specific languanges for visualization, and flow visualization.

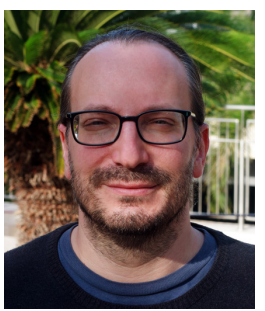

Markus Hadwiger received his $\mathrm{PhD}$ in computer science from Vienna University of Technology in 2004. He worked as a senior researcher at the VRVis Research Center in Vienna from 2000 to 2009. He was an assistant professor from 2009 to 2014 and then an associate professor (up to present) at KAUST, Saudi Arabia. He is interested in scientific visualization and visual computing, specifically on extreme-scale volume and flow visualization, multiresolution techniques, and GPU algorithms and architecture. 